Pacific

Journal of

Mathematics

\title{
THE LANDAU-LIFSHITZ-MAXWELL EQUATION
} IN DIMENSION THREE

\author{
ShiJin Ding, Xiangao LiU AND Changyou Wang
}

Volume $243 \quad$ No. 2

December 2009 


\title{
THE LANDAU-LIFSHITZ-MAXWELL EQUATION IN DIMENSION THREE
}

\author{
ShiJin Ding, Xiangao LiU And Changyou Wang
}

\begin{abstract}
In dimension three, we show the existence of weak solutions $(u, H, E)$ to the Landau-Lifshitz equation coupled with the time-dependent Maxwell equation such that $u$ is Hölder continuous away from a closed set $\Sigma$ that has locally finite 3-dimensional parabolic Hausdorff measure. For two reduced Maxwell equations, Hölder continuity of $\nabla u$ away from $\Sigma$ is also established.
\end{abstract}

\section{Introduction}

For a bounded, smooth domain $\Omega \subseteq \mathbb{R}^{3}$, we consider the Landau-Lifshitz-Maxwell equation:

$$
\begin{array}{ll}
\frac{\partial u}{\partial t}=\beta_{1} u \times(\Delta u+H)-\beta_{2} u \times(u \times(\Delta u+H)) & \text { in } \Omega \times \mathbb{R}_{+}, \\
\nabla \times H=\varepsilon_{0} \frac{\partial E}{\partial t}+\sigma E & \text { in } \mathbb{R}^{3} \times \mathbb{R}_{+}, \\
\nabla \times E=-\frac{\partial}{\partial t}(H+\beta \bar{u}) & \text { in } \mathbb{R}^{3} \times \mathbb{R}_{+},
\end{array}
$$

where $u: \Omega \times \mathbb{R}_{+} \rightarrow S^{2}$ is the magnetization field, $H: \mathbb{R}^{3} \times \mathbb{R}_{+} \rightarrow \mathbb{R}^{3}$ is the magnetic field, $E: \mathbb{R}^{3} \times \mathbb{R}_{+} \rightarrow \mathbb{R}^{3}$ is the electric field, $H^{e} \equiv \triangle Z+H$ is the effective magnetic field, $\beta_{1}$ is the gyromagnetic coefficient, $\beta_{2} \geq 0$ is the Gilbert damping coefficient, $\varepsilon_{0} \geq 0, \sigma \geq 0$ is the conductivity constant, $\beta$ is the magnetic permeability of free space, and $\bar{u}$ is an extension of $u$ such that $\bar{u}=0$ outside $\Omega$. The system (1-1)-(1-3) was originally proposed by Landau and Lifshitz [1935] to model the dynamics of magnetization, magnetic field and electric field for the ferromagnetic materials.

The coupled Maxwell equations (1-2) and (1-3) can be written as

$$
\frac{\partial B}{\partial t}=-\nabla \times E \quad \text { and } \quad \frac{\partial D}{\partial t}+\sigma E=\nabla \times H \quad \text { in } \mathbb{R}^{3} \times \mathbb{R}_{+},
$$

MSC2000: primary 35K55; secondary 35B65.

Keywords: Landau-Lifshitz-Gilbert equation, time-dependent Maxwell equation.

Ding is partially supported by the NSFC Grant 10471050, National 973 Program of China (No. 2006CB805902), Guangdong Provincial Natural Science Foundation (No. 7005795), and by University Special Research Foundation for Ph.D. Program (No. 20060574002). Liu is partially supported by NSFC 10631020 . Wang is partially supported by NSF 0601162. 
where $D$ and $B$ are the electric and magnetic displacements, given by

$$
D=\varepsilon_{0} E, \quad B=H+\beta \bar{u} \quad \text { in } \mathbb{R}^{3} \times \mathbb{R}_{+} .
$$

Note that when $H=E=0$ and $\beta=0$, the system (1-1)-(1-3) reduces to the Landau-Lifshitz-Gilbert equation for $Z: \Omega \times \mathbb{R}_{+} \rightarrow S^{2}$ :

$$
\frac{\partial Z}{\partial t}=\beta_{1} Z \times \triangle Z-\beta_{2} Z \times(Z \times \triangle Z) .
$$

Equation (1-6) is a hybrid between the Schrödinger flow into $S^{2}(\partial u / \partial t=u \times \Delta u$ for $\left.\beta_{2}=0\right)$ and the heat flow of a harmonic map into $S^{2}\left(\partial u / \partial t=\Delta u+|\nabla u|^{2} u\right.$ for $\beta_{1}=0$ ). There have been many works on both the existence and regularity of weak solutions to (1-6) in recent years. Zhou and Guo [1987] proved the existence of global weak solutions of (1-6) under suitable initial-boundary conditions. The unique smooth solution of (1-6) in dimension one was established in [Zhou et al. 1991]. Alouges and Soyeur [1992] proved that given $0<\beta_{2}$ and the initial data $u_{0}: \mathbb{R}^{3} \rightarrow S^{2}$ with $\nabla u_{0} \in L^{2}\left(\mathbb{R}^{3}\right)$, there exists a global weak solution of (1-6) in $\mathbb{R}^{3}$. Moreover, if $u_{0} \in H^{1}(\Omega)$ and $\beta_{2}>0$, the Neumann boundary value problem of (1-6) in a bounded domain $\Omega \subset \mathbb{R}^{3}$ may admit infinitely many weak solutions. For regularity of weak solutions to (1-6), Guo and Hong [1993] established the existence of a global, weak solution with finitely many singular points in dimension two, and Chen, Ding and Guo [1998] proved the uniqueness of weak solutions whose energies are nonincreasing in time at dimension two. In dimension three, Melcher [2005] proved the existence of global weak solutions to (1-6) for $\Omega=\mathbb{R}^{3}$, which are smooth away from a closed set of locally finite 3-dimensional parabolic Hausdorff measure. Later, Wang [2006] established the existence of partially smooth weak solutions to (1-6) in any bounded domain $\Omega$ of dimension at most 4 . It is unknown whether these results of Melcher and Wang can be extended to higher dimensions. It is also an interesting question to study the regularity of suitable weak solutions to (1-6). Moser [2002] proved, in dimensions $n \leq 4$, a partial regularity theorem of weak solutions to (1-6) that are stationary, a notion analogous to that of heat flow of harmonic maps introduced in [Feldman 1994; Chen et al. 1995; Chen and Wang 1996] (see also [2004]). More recently, Ding and Wang [2007] proved that shorttime, smooth solutions to (1-6) may develop a finite-time singularity in dimensions 3 and 4 for suitable initial-boundary data.

Motivated by these studies of (1-6), we are interested in the Landau-Lifshitz system coupled with the time-dependent Maxwell equations (1-1)-(1-3).

There has been some work on the system (1-1)-(1-3). Guo and Su [1997] used Galerkin's method to establish the existence of global, weak solutions with periodic initial conditions in dimension three. Carbou and Fabrie [1998] used the GinzburgLandau approximation scheme to show the existence of global, weak solutions to 
the system (1-1)-(1-3) under the Neumann boundary condition in dimension three, and studied the long-time behavior of the weak solution by the method of time average. See also [Joly et al. 2000; Ding et al. 2007] for related results.

The regularity issue of the system (1-1)-(1-3) is a challenging problem. There are very few results in the literature. Ding and Guo [2004] proved a partial regularity theorem for stationary solutions to the Landau-Lifshitz equation (1-1) coupled with the quasistationary Maxwell equation

$$
\operatorname{div}(H+\beta \bar{u})=0 \quad \text { and } \quad \nabla \times H=0 \text { in } \mathscr{D}^{\prime}\left(\mathbb{R}^{3}\right) .
$$

By modifying the techniques by [Wang 2006], Ding and Guo [2008] proved the existence of partially smooth weak solutions to (1-1)+(1-7) in dimension three.

There is an essential difference between (1-7) and (1-2)-(1-3): the former is elliptic and $H \in \bigcap_{p>1} L^{p}\left(\mathbb{R}^{3}, \mathbb{R}^{3}\right)$, while (1-2)-(1-3) is a hyperbolic system and the regularity for $H(\cdot, t)$ and $E(\cdot, t)$ is no better than that of $H(\cdot, 0)$ and $E(\cdot, 0)$. The hyperbolicity of (1-2) and (1-3) imposes serious difficulties to studying the regularity of (1-1).

In this paper, we establish the existence of partially regular, weak solutions of the Landau-Lifshitz-Maxwell system (1-1)-(1-3) with respect to the following initial-boundary conditions:

$$
\begin{aligned}
\frac{\partial u}{\partial v} & =0 & & \text { on } \Omega \times \mathbb{R}_{+}, \\
u(x, 0) & =u_{0}(x) & & \text { in } \Omega, \\
H(x, 0) & =H_{0}(x) & & \text { in } \mathbb{R}^{3}, \\
E(x, 0) & =E_{0}(x) & & \text { in } \mathbb{R}^{3} .
\end{aligned}
$$

We assume throughout the paper that

$$
\left|u_{0}\right|=1 \text { a.e. in } \Omega, \quad H_{0} \in L^{2}\left(\mathbb{R}^{3}, \mathbb{R}^{3}\right), \quad E_{0} \in L^{2}\left(\mathbb{R}^{3}, \mathbb{R}^{3}\right) .
$$

For convenience, we study an equivalent form of the Landau-Lifshitz-Maxwell equation (1-1) (see [Guo and Hong 1992; 1993]):

$$
\alpha_{1} \frac{\partial u}{\partial t}+\alpha_{2} u \times \frac{\partial u}{\partial t}=\left(\Delta u+|\nabla u|^{2} u\right)+(H-\langle H, u\rangle u) \quad \text { in } \Omega \times \mathbb{R}_{+},
$$

where $\alpha_{1}, \alpha_{2} \in \mathbb{R}$ represent suitable normalizations of $\beta_{2}$ and $\beta_{1}$ such that

$$
0<\alpha_{1}<1, \quad \alpha_{1}^{2}+\alpha_{2}^{2}=1 .
$$

Definition 1.1. The triple $(u, H, E)$ is a weak solution of the system (1-13)+(1-2)(1-3) with the initial-boundary conditions (1-8)-(1-11) if

(i) $u \in L_{\mathrm{loc}}^{\infty}\left(\mathbb{R}_{+}, H^{1}\left(\Omega, S^{2}\right)\right), \partial u / \partial t \in L_{\mathrm{loc}}^{2}\left(\Omega \times \mathbb{R}_{+}\right)$and $H, E \in L_{\mathrm{loc}}^{\infty}\left(\mathbb{R}_{+}, L^{2}\left(\mathbb{R}^{3}\right)\right)$; 
(ii) $u$ satisfies (1-13) in the distribution sense, that is, for any $\Phi \in C^{\infty}\left(\Omega \times \mathbb{R}_{+}, \mathbb{R}^{3}\right)$ with $\Phi(\cdot, 0)=\Phi(\cdot,+\infty)=0$,

$$
\begin{aligned}
\int_{\Omega \times \mathbb{R}_{+}}\left(\alpha_{1} \frac{\partial u}{\partial t}+\alpha_{2} u \times \frac{\partial u}{\partial t}\right) \cdot \Phi=\int_{\Omega \times \mathbb{R}_{+}} & \left.-\nabla u \cdot \nabla \Phi+|\nabla u|^{2} u \cdot \Phi\right) \\
& +\int_{\Omega \times \mathbb{R}_{+}}(H-\langle H, u\rangle u) \cdot \Phi
\end{aligned}
$$

and $u(\cdot, 0)=u_{0}$ in the sense of trace;

(iii) for any $\Phi \in C^{\infty}\left(\mathbb{R}^{3} \times \mathbb{R}_{+}, \mathbb{R}^{3}\right)$ with $\Phi(\cdot,+\infty)=0$,

$(1-15)-\int_{\mathbb{R}^{3} \times \mathbb{R}_{+}}\left(\varepsilon_{0} E \cdot \frac{\partial \Phi}{\partial t}+H \cdot \nabla \times \Phi\right)+\sigma \int_{\mathbb{R}^{3} \times \mathbb{R}_{+}} E \cdot \Phi=\varepsilon_{0} \int_{\mathbb{R}^{3}} E_{0}(x) \cdot \Phi(x, 0) ;$

(iv) for any $\Phi \in C^{\infty}\left(\mathbb{R}^{3} \times \mathbb{R}_{+}, \mathbb{R}^{3}\right)$ with $\Phi(\cdot,+\infty)=0$,

$$
\begin{aligned}
-\int_{\mathbb{R}^{3} \times \mathbb{R}_{+}}(H+\beta \bar{u}) \cdot \frac{\partial \Phi}{\partial t}+ & \int_{\mathbb{R}^{3} \times \mathbb{R}_{+}} E \cdot \nabla \times \Phi \\
& =\beta \int_{\Omega} u_{0}(x) \cdot \Phi(x, 0)+\int_{\mathbb{R}^{3}} H_{0}(x) \cdot \Phi(x, 0) .
\end{aligned}
$$

To state our results, we also need some notation. For $z_{0}=\left(x_{0}, t_{0}\right) \in \mathbb{R}^{3} \times \mathbb{R}$ and $r>0$, set

$$
B_{r}\left(x_{0}\right)=\left\{x \in \mathbb{R}^{3}:\left|x-x_{0}\right|<r_{0}\right\} \quad \text { and } \quad P_{r}\left(z_{0}\right)=B_{r}\left(x_{0}\right) \times\left(t_{0}-r^{2}, t_{0}\right) .
$$

For any subset $D \subset \mathbb{R}^{4}$, the three-dimensional parabolic Hausdorff measure, $\mathscr{P}^{3}(D)$, is defined by

$$
\mathscr{P}^{3}(D)=\lim _{\delta \downarrow 0}\left(\inf \left\{\sum_{i=1}^{\infty} r_{i}^{3}: D \subset \bigcup_{i=1}^{\infty} P_{r_{i}}\left(z_{i}\right), 0<r_{i} \leq \delta\right\}\right) .
$$

We say a subset $D \subset \mathbb{R}^{4}$ has locally finite three-dimensional parabolic Hausdorff measure if

$$
\mathscr{P}^{3}\left(D \cap P_{R}(0)\right)<+\infty \quad \text { for all } R>0 .
$$

Theorem 1.2. For any $u_{0} \in H^{1}\left(\Omega, S^{2}\right), H_{0} \in L^{2}\left(\mathbb{R}^{3}, \mathbb{R}^{3}\right)$ and $E_{0} \in L^{2}\left(\mathbb{R}^{3}, \mathbb{R}^{3}\right)$, there exists a global weak solution $(u, H, E)$ to the Landau-Lifshitz-Maxwell system (1-13)+(1-2)-(1-3) under the initial-boundary conditions (1-8)-(1-11) such that there exists a closed subset $\Sigma \subset \Omega \times \mathbb{R}_{+}$having locally finite 3-dimensional parabolic Hausdorff measure and satisfying $u \in C^{1 / 2}\left(\Omega \times \mathbb{R}_{+} \backslash \Sigma, S^{2}\right)$.

To study the higher-order regularity of weak solutions to (1-13)+(1-2)-(1-3) obtained by Theorem 1.2, we restrict to two special cases: 
(i) The constant $\varepsilon_{0}$ vanishes in (1-2), and (1-2)-(1-3) become

$$
\nabla \times(\nabla \times H)=-\sigma \frac{\partial}{\partial t}(H+\beta \bar{u}) \quad \text { in } \mathbb{R}^{3} .
$$

(ii) The constant $\beta$ vanishes in (1-3), and (1-2)-(1-3) become

$$
\nabla \times H=\varepsilon_{0} \frac{\partial E}{\partial t}+\sigma E, \quad \nabla \times E=-\frac{\partial H}{\partial t} \quad \text { in } \mathbb{R}^{3} .
$$

Theorem 1.3. Assume that $\delta>0$ is a constant. For any $u_{0} \in H^{1}\left(\Omega, S^{2}\right)$ and $H_{0} \in$ $H^{1}\left(\mathbb{R}^{3}, \mathbb{R}^{3}\right)$ satisfying $\nabla \cdot\left(H_{0}+\beta \bar{u}_{0}\right)=0$ in $\mathscr{D}^{\prime}\left(\mathbb{R}^{3}\right)$, there exists a weak solution $(u, H)$ of the Landau-Lifshitz system (1-13) coupled with (1-17) under the initialboundary conditions (1-8)-(1-10) such that $H \in \bigcap_{T>0} H^{1}\left(\mathbb{R}^{3} \times[0, T], \mathbb{R}^{3}\right)$ and there exists a closed subset $\Sigma \subset \Omega \times \mathbb{R}_{+}$of locally finite 3-dimensional parabolic Hausdorff measure and satisfying $\nabla u \in C^{\alpha}\left(\Omega \times \mathbb{R}_{+} \backslash \Sigma\right)$ for some $0<\alpha<1$, and $\nabla^{2} u, \partial u / \partial t \in L_{\text {loc }}^{6}\left(\Omega \times \mathbb{R}_{+} \backslash \Sigma\right)$.

Theorem 1.4. For any $u_{0} \in H^{1}\left(\Omega, S^{2}\right)$ and $H_{0}, E_{0} \in H^{1}\left(\mathbb{R}^{3}, \mathbb{R}^{3}\right)$ that satisfy $\nabla \cdot H_{0}=\nabla \cdot E_{0}=0$ in $\mathscr{D}^{\prime}\left(\mathbb{R}^{3}\right)$, there exists a weak solution $(u, H, E)$ of the Landau-Lifshitz system (1-13) coupled with (1-18) under the initial-boundary conditions (1-8)-(1-11) such that $\partial H / \partial t, \partial E / \partial t \in L_{\mathrm{loc}}^{\infty}\left(\mathbb{R}_{+}, L^{2}\left(\mathbb{R}^{3}\right)\right)$, and there exists a closed subset $\Sigma \subset \Omega \times \mathbb{R}_{+}$having locally finite 3-dimensional parabolic Hausdorff measure and satisfying $\nabla u \in C^{\alpha}\left(\Omega \times \mathbb{R}_{+} \backslash \Sigma\right)$ for some $0<\alpha<1$, and $\nabla^{2} u, \partial u / \partial t \in L_{\text {loc }}^{6}\left(\Omega \times \mathbb{R}_{+} \backslash \Sigma\right)$.

The ideas to approach these theorems are based on an analysis of the GinzburgLandau approximate equation: for $\varepsilon>0$,

$$
\alpha_{1} \frac{\partial u^{\varepsilon}}{\partial t}+\alpha_{2} u^{\varepsilon} \times \frac{\partial u^{\varepsilon}}{\partial t}=\Delta u^{\varepsilon}+\frac{1}{\varepsilon^{2}}\left(1-\left|u^{\varepsilon}\right|^{2}\right) u^{\varepsilon}+u^{\varepsilon} \times\left(H^{\varepsilon} \times u^{\varepsilon}\right) \text { in } \Omega \times \mathbb{R}_{+}
$$

By using an argument similar to the one in [Ding and Wang 2007], it is not hard to see that the corresponding partial regularity property at the boundary also holds for the weak solution obtained in Theorems 1.2, 1.3, and 1.4. For example, Theorem 1.2 can be extended so that there exists a closed subset $\Sigma_{1} \subseteq \partial \Omega$, with $\mathscr{P}^{3}\left(\Sigma_{1}\right)<$ $+\infty$, such that $u \in C^{1 / 2}\left(\bar{\Omega} \backslash\left(\Sigma \cup \Sigma_{1}\right), S^{2}\right)$.

The paper is written as follows. In Section 2, we establish a uniform energy estimate for (1-19). In Section 3, we sketch the time slice monotonicity. In Section 4 we establish a lower bound estimate of solutions to (1-19). In Section 5, we obtain the decay estimate of solutions to (1-19) under the smallness condition and prove Theorem 1.2. In Section 6, we establish a partial $C^{\alpha}$-regularity of $\nabla u$ and prove both Theorems 1.3 and 1.4. 


\section{Estimate of the energy in (1-19)}

In this section, we sketch the existence of global weak solutions to (1-19)+(1-2)(1-3), associated with (1-8)-(1-11) by Galerkin's method and their corresponding energy estimates. Here we modify an argument from [Carbou and Fabrie 1998] to handle (1-19). Note the difference between (1-19) and the approximate equation employed by that paper: we approximate $H-\langle H, u\rangle u$ in (1-13) by $u^{\varepsilon} \times\left(H^{\varepsilon} \times u^{\varepsilon}\right)$ in (1-19), while Carbou and Fabrie approximate the same term in (1-13) by $H^{\varepsilon}$. An advantage of our approximation is that we have the upper bound $\left|u^{\varepsilon}\right| \leq 1$, which plays a crucial role in establishing a priori continuity estimates for $u^{\varepsilon}$, and hence the existence of partially smooth solutions, while theirs yields an optimal energy inequality [Carbou and Fabrie 1998, page 387, (2.12)], which is important in their study of long-time behaviors by the method of time average.

We begin with a general $L^{\infty}$-estimate of weak solutions $u^{\varepsilon}$ to (1-19).

Lemma 2.1. For $\varepsilon>0$, assume $u_{0} \in H^{1}\left(\Omega, S^{2}\right), H_{0} \in L^{2}\left(\mathbb{R}^{3}, \mathbb{R}^{3}\right)$ and $E_{0} \in$ $L^{2}\left(\mathbb{R}^{3}, \mathbb{R}^{3}\right)$. Let $\left(u^{\varepsilon}, H^{\varepsilon}, E^{\varepsilon}\right)$ be any weak solution of $(1-19)+(1-2)-(1-3)$ under conditions (1-8)-(1-11). Then $\left|u^{\varepsilon}\right|(x, t) \leq 1$ for any $(x, t) \in \Omega \times \mathbb{R}_{+}$.

Proof. Multiplying (1-19) by $u^{\varepsilon}$ and using the equalities $u^{\varepsilon} \cdot u^{\varepsilon} \times\left(\partial u^{\varepsilon} / \partial t\right)=0$ and $u^{\varepsilon} \cdot u^{\varepsilon} \times\left(H^{\varepsilon} \times u^{\varepsilon}\right)=0$, we have

$$
\left(\alpha_{1} \frac{\partial}{\partial t}-\Delta\right)\left(\left|u^{\varepsilon}\right|^{2}-1\right)=-2\left(\left|\nabla u^{\varepsilon}\right|^{2}+\frac{1}{\varepsilon^{2}}\left(\left|u^{\varepsilon}\right|^{2}-1\right)\left|u^{\varepsilon}\right|^{2}\right)
$$

hence

$$
\left(\alpha_{1} \frac{\partial}{\partial t}-\Delta\right)\left(\left|u^{\varepsilon}\right|^{2}-1\right)_{+} \leq 0 \quad \text { in } \Omega \times \mathbb{R}_{+},
$$

where $\left(\left|u^{\varepsilon}\right|^{2}-1\right)_{+}$is the positive part of $\left(\left|u^{\varepsilon}\right|^{2}-1\right)$. The conclusion now holds by the weak maximum principle of the heat equation [Lieberman 1996].

Now we sketch the existence of weak solutions to (1-19) that enjoy energy estimates by Galerkin's method. We borrow some notation from [Carbou and Fabrie 1998, pages 388-395]. Let $\left\{\phi_{k}\right\}_{k} \subseteq H^{2}(\Omega)$ be eigenfunctions of $\Delta$ with zero Neumann boundary condition that form an orthonormal basis in $L^{2}(\Omega)$ and an orthogonal basis in $H^{1}(\Omega)$ and $H^{2}(\Omega)$. For $1 \leq N<+\infty$, set $V_{N}=\operatorname{span}\left\{\phi_{1}, \ldots, \phi_{N}\right\}$. Define

$$
\mathbb{W}_{\text {curl }}\left(\mathbb{R}^{3}\right)=\left\{\psi \in L^{2}\left(\mathbb{R}^{3}, \mathbb{R}^{3}\right), \nabla \times \psi \in L^{2}\left(\mathbb{R}^{3}, \mathbb{R}^{3}\right)\right\} .
$$

Let $\left\{\psi_{k}\right\}_{k}$ be an orthogonal basis of $\mathbb{H}_{\text {curl }}\left(\mathbb{R}^{3}\right)$ that is orthonormal in $L^{2}\left(\mathbb{R}^{3}\right)$ and $W_{N}=\operatorname{span}\left\{\psi_{1}, \ldots, \psi_{N}\right\}$. Denote by $\Pi_{V_{N}}: L^{2}(\Omega) \rightarrow V_{N}$, and $\Pi_{W_{N}}: L^{2}(\Omega) \rightarrow W_{N}$ the orthogonal projections. Define the retraction map $\Pi: \mathbb{R}^{3} \rightarrow B_{1}$ by setting

$$
\Pi(p)= \begin{cases}p & \text { if }|p| \leq 1 \\ p /|p| & \text { if }|p|>1\end{cases}
$$


Define functions $u_{N} \in V_{N}$ and $H_{N}, E_{N} \in W_{N}$ by $u_{N}(x, t)=\sum_{k=1}^{N} v_{k}(t) \phi_{k}(x), H_{N}(x, t)=\sum_{k=1}^{N} h_{k}(t) \psi_{k}(x), E_{N}(x, t)=\sum_{k=1}^{N} e_{k}(t) \psi_{k}(x)$, satisfying, for all $\Phi \in V_{N}$ and $\Psi \in W_{N}$,

$$
\begin{aligned}
\int_{\Omega}\left(\alpha_{1} \frac{\partial u_{N}}{\partial t}+\right. & \left.\alpha_{2} u_{N} \times \frac{\partial u_{N}}{\partial t}\right) \cdot \Phi \\
=\int_{\Omega}\left(-\nabla u_{N} \cdot \nabla \Phi+\frac{1}{\varepsilon^{2}}\left(1-\left|u_{N}\right|^{2}\right) u_{N} \cdot \Phi\right) & \quad+\int_{\Omega} \Pi\left(u_{N}\right) \times\left(H_{N} \times \Pi\left(u_{N}\right)\right) \cdot \Phi
\end{aligned}
$$

$$
\begin{gathered}
\int_{\mathbb{R}^{3}}\left(\varepsilon_{0} \frac{\partial E_{N}}{\partial t}+\sigma E_{N}\right) \cdot \Psi=\int_{\mathbb{R}^{3}} H_{N} \cdot(\nabla \times \Psi), \\
\int_{\mathbb{R}^{3}} \frac{\partial}{\partial t}\left(H_{N}+\beta \bar{u}_{N}\right) \cdot \Psi=-\int_{\mathbb{R}^{3}} E_{N} \cdot(\nabla \times \Psi),
\end{gathered}
$$

under the initial conditions

$$
\left.u_{N}\right|_{t=0}=\Pi_{V_{N}}\left(u_{0}\right),\left.\quad H_{N}\right|_{t=0}=\Pi_{W_{N}}\left(H_{0}\right),\left.\quad E_{N}\right|_{t=0}=\Pi_{W_{N}}\left(E_{0}\right) .
$$

Throughout this section, we will use the following fact:

$$
\lim _{N \rightarrow \infty} \int_{\Omega} e_{\varepsilon}\left(u_{N}(0)\right)=\int_{\Omega} \frac{1}{2}\left|\nabla u_{0}\right|^{2} .
$$

Note that (2-3)-(2-6) reduces to a system of first order ODEs for $\left(v_{k}, h_{k}, e_{k}\right)_{k}$. Moreover, since $P\left(u_{N}\right)(v)=\alpha_{1} v+\alpha_{2} u_{N} \times v: \mathbb{R}^{3} \rightarrow \mathbb{R}^{3}$ is one-to-one, we can solve (2-3) for the derivative in time. Hence there exists a local solution $\left(u_{N}, H_{N}, E_{N}\right)$ of (2-3)-(2-6). The following uniform estimate shows that $\left(u_{N}, H_{N}, E_{N}\right)$ is also global in time and converges to a global weak solution of (1-19)+(1-2)-(1-3).

Lemma 2.2. For $\varepsilon>0$, assume $u_{0} \in H^{1}\left(\Omega, S^{2}\right), H_{0} \in L^{2}\left(\mathbb{R}^{3}, \mathbb{R}^{3}\right)$ and $E_{0} \in$ $L^{2}\left(\mathbb{R}^{3}, \mathbb{R}^{3}\right)$. Then there exists a global weak solution $\left(u^{\varepsilon}, H^{\varepsilon}, E^{\varepsilon}\right)$ to $(1-19)+(1-2)-$ (1-3) under conditions (1-8)-(1-11), such that for any $0<T<+\infty$ we have

$$
\sigma \int_{0}^{T} \int_{\mathbb{R}^{3}}\left|E^{\varepsilon}\right|^{2}+\alpha_{1} \int_{0}^{T} \int_{\Omega}\left|\frac{\partial u^{\varepsilon}}{\partial t}\right|^{2}+\mathscr{E}_{\varepsilon}(T) \leq e^{C T \mathscr{E}_{0}}
$$

where $C>0$ depends only on $\beta$ and $\alpha_{1}$, and

$$
\begin{aligned}
\mathscr{E}_{0} & =\int_{\Omega} \frac{1}{2}\left|\nabla u_{0}\right|^{2}+\int_{\mathbb{R}^{3}}\left(\frac{1}{2} \varepsilon_{0}\left|E_{0}\right|^{2}+\frac{1}{2}\left|H_{0}\right|^{2}\right), \\
\mathscr{E}_{\varepsilon}(t) & =\int_{\Omega} e_{\varepsilon}\left(u^{\varepsilon}(t)\right)+\int_{\mathbb{R}^{3}}\left(\frac{1}{2} \varepsilon_{0}\left|E^{\varepsilon}(t)\right|^{2}+\frac{1}{2}\left|H^{\varepsilon}(t)\right|^{2}\right)
\end{aligned}
$$


with $e_{\varepsilon}\left(u^{\varepsilon}(t)\right)=\frac{1}{2}\left|\nabla u^{\varepsilon}(t)\right|^{2}+\frac{1}{4 \varepsilon^{2}}\left(1-\left|u^{\varepsilon}(t)\right|^{2}\right)^{2}$.

Proof. We first establish the estimate (2-8) for Galerkin's approximate solutions $\left(u_{N}, H_{N}, E_{N}\right)$. Then we employ this estimate to extract a subsequence that converges to a global weak solution $\left(u^{\varepsilon}, H^{\varepsilon}, E^{\varepsilon}\right)$ to (1-19)+(1-2)-(1-3).

Testing (2-3) with $\Phi=\partial u_{N} / \partial t$ and integrating over $\Omega$ gives

$$
\begin{aligned}
\int_{\Omega} \alpha_{1}\left|\frac{\partial u_{N}}{\partial t}\right|^{2}+\frac{d}{d t} \int_{\Omega} e_{\varepsilon}\left(u_{N}\right) & =\int_{\Omega} \Pi\left(u_{N}\right) \times\left(H_{N} \times \Pi\left(u_{N}\right)\right) \cdot \frac{\partial u_{N}}{\partial t} \\
& \leq \int_{\Omega}\left|H_{N}\right|\left|\frac{\partial u_{N}}{\partial t}\right|,
\end{aligned}
$$

where we used the inequalities $\left|\Pi\left(u_{N}\right)\right| \leq 1$ and $\left|\Pi\left(u_{N}\right) \times\left(H_{N} \times \Pi\left(u_{N}\right)\right)\right| \leq\left|H_{N}\right|$.

Testing (2-4) with $\Psi=E_{N}$ and integrating over $\mathbb{R}^{3}$ gives

$$
\int_{\mathbb{R}^{3}} \nabla \times H_{N} \cdot E_{N}=\frac{d}{d t} \int_{\mathbb{R}^{3}} \frac{1}{2} \varepsilon_{0}\left|E_{N}\right|^{2}+\int_{\mathbb{R}^{3}} \sigma\left|E_{N}\right|^{2} .
$$

Testing (2-5) with $\Psi=H_{N}$ and integrating over $\mathbb{R}^{3}$ gives

$$
-\int_{\mathbb{R}^{3}} \nabla \times E_{N} \cdot H_{N}=\frac{d}{d t} \int_{\mathbb{R}^{3}} \frac{1}{2}\left|H_{N}\right|^{2}+\beta \int_{\Omega} H_{N} \cdot \frac{\partial u_{N}}{\partial t} .
$$

Adding together (2-11) and (2-12), and using the identity

$$
\int_{\mathbb{R}^{3}}\left(\nabla \times H_{N} \cdot E_{N}-\nabla \times E_{N} \cdot H_{N}\right)=0,
$$

we obtain

$$
\begin{aligned}
\frac{d}{d t} \int_{\mathbb{R}^{3}}\left(\frac{1}{2} \varepsilon_{0}\left|E_{N}\right|^{2}+\frac{1}{2}\left|H_{N}\right|^{2}\right)+\sigma \int_{\mathbb{R}^{3}}\left|E_{N}\right|^{2} & =-\beta \int_{\Omega} H_{N} \cdot \frac{\partial u_{N}}{\partial t} \\
& \leq \beta \int_{\Omega}\left|H_{N}\right|\left|\frac{\partial u_{N}}{\partial t}\right| .
\end{aligned}
$$

Adding (2-10) and (2-13) together gives

$$
\begin{array}{r}
\sigma \int_{\mathbb{R}^{3}}\left|E_{N}\right|^{2}+\alpha_{1} \int_{\Omega}\left|\frac{\partial u_{N}}{\partial t}\right|^{2}+\frac{d}{d t}\left(\int_{\Omega} e_{\varepsilon}\left(u_{N}\right)+\int_{\mathbb{R}^{3}}\left(\frac{1}{2} \varepsilon_{0}\left|E_{N}\right|^{2}+\frac{1}{2}\left|H_{N}\right|^{2}\right)\right) \\
\leq(1+\beta) \int_{\Omega}\left|H_{N}\right|\left|\frac{\partial u_{N}}{\partial t}\right| \leq \frac{\alpha_{1}}{4} \int_{\Omega}\left|\frac{\partial u_{N}}{\partial t}\right|^{2}+\frac{(1+\beta)^{2}}{\alpha_{1}} \int_{\mathbb{R}^{3}}\left|H_{N}\right|^{2},
\end{array}
$$

where we used the Cauchy-Schwarz inequality in the last step. Using Grönwall's inequality in (2-14) and integrating from $t=0$ to $t=T$ gives 
$(2-15)$

$$
\begin{aligned}
\sigma \int_{0}^{T} \int_{\mathbb{R}^{3}}\left|E_{N}\right|^{2}+\alpha_{1} \int_{0}^{T} \int_{\Omega}\left|\frac{\partial u_{N}}{\partial t}\right|^{2}+\left(\int_{\Omega} e_{\varepsilon}\left(u_{N}\right)+\int_{\mathbb{R}^{3}}\left(\frac{1}{2} \varepsilon_{0}\left|E_{N}\right|^{2}+\frac{1}{2}\left|H_{N}\right|^{2}\right)\right)(T) \\
\leq e^{C T}\left(\int_{\Omega} e_{\varepsilon}\left(u_{N}\right)+\int_{\mathbb{R}^{3}}\left(\frac{1}{2} \varepsilon_{0}\left|E_{N}\right|^{2}+\frac{1}{2}\left|H_{N}\right|^{2}\right)\right)(0) \\
\leq e^{C T}\left(o(1)+\int_{\Omega} e_{\varepsilon}\left(u_{0}\right)+\int_{\mathbb{R}^{3}}\left(\frac{1}{2} \varepsilon_{0}\left|E_{0}\right|^{2}+\frac{1}{2}\left|H_{0}\right|^{2}\right)\right) \\
=e^{C T}\left(\mathscr{E}_{0}+o(1)\right) .
\end{aligned}
$$

Here we have used (2-7) and $o(1)$ denotes a quantity that tends to 0 as $N$ tends to $\infty$. It follows from the bound (2-15) that there exists a subsequence of $\left(u_{N}, H_{N}, E_{N}\right)$, still written the same, such that for any $0<T<+\infty$,

$$
\begin{gathered}
u_{N} \rightarrow u^{\varepsilon} \text { weak }^{*} \text { in } L^{\infty}\left([0, T], H^{1}(\Omega)\right), \quad \frac{\partial u_{N}}{\partial t} \rightarrow \frac{\partial u^{\varepsilon}}{\partial t} \text { in } L^{2}(\Omega \times[0, T]), \\
E_{N} \rightarrow E^{\varepsilon}, H_{N} \rightarrow H^{\varepsilon} \text { weak }^{*} \text { in } L^{\infty}\left([0, T], L^{2}\left(\mathbb{R}^{3}\right)\right) .
\end{gathered}
$$

By Aubin's Lemma (see also [Carbou and Fabrie 1998]),

$$
u_{N} \rightarrow u^{\varepsilon} \text { strongly in } L^{4}(\Omega \times[0, T]) .
$$

Since $\left|\Pi\left(u_{N}\right)\right| \leq\left|u_{N}\right|$ and $\int_{\Omega}\left|\nabla\left(\Pi\left(u_{N}\right)\right)\right|^{2} \leq \int_{\Omega}\left|\nabla u_{N}\right|^{2}$, we also have

$$
\Pi\left(u_{N}\right) \rightarrow \Pi\left(u^{\varepsilon}\right) \text { strongly in } L^{4}(\Omega \times[0, T]) .
$$

It is readily seen that (2-15) implies that $\left(u^{\varepsilon}, H^{\varepsilon}, E^{\varepsilon}\right)$ satisfies (2-8) and the initial conditions (1-8)-(1-11). It is also not hard to see that $\left(H^{\varepsilon}, E^{\varepsilon}\right)$ are weak solutions to (1-2)-(1-3). Similarly to [Carbou and Fabrie 1998, page 392], we can check that

$$
\alpha_{1} \frac{\partial u^{\varepsilon}}{\partial t}+\alpha_{2} u^{\varepsilon} \times \frac{\partial u^{\varepsilon}}{\partial t}=\Delta u^{\varepsilon}+\frac{1}{\varepsilon^{2}}\left(1-\left|u^{\varepsilon}\right|^{2}\right) u^{\varepsilon}+\Pi\left(u^{\varepsilon}\right) \times\left(H^{\varepsilon} \times \Pi\left(u^{\varepsilon}\right)\right) .
$$

Multiplying (2-16) by $u^{\varepsilon}$ and observing that $\Pi\left(u^{\varepsilon}\right) \times\left(H^{\varepsilon} \times \Pi\left(u^{\varepsilon}\right)\right) \cdot u^{\varepsilon}=0$, we see that $u^{\varepsilon}$ satisfies (2-1). Hence Lemma 2.1 implies that $\left|u^{\varepsilon}\right| \leq 1$. Thus $\Pi\left(u^{\varepsilon}\right)=u^{\varepsilon}$ and (2-16) yields (1-19).

In order to establish a partial $C^{\alpha}$-regularity of $\nabla u$ for weak solutions $u$ to (1-13) coupled with the Maxwell equations (1-17) or (1-18), we need uniform estimates of $H^{\varepsilon}, E^{\varepsilon}$ in $H_{\mathrm{loc}}^{1}\left(\mathbb{R}^{3} \times \mathbb{R}_{+}\right)$. More precisely:

Lemma 2.3. Suppose that $u_{0} \in H^{1}\left(\Omega, S^{2}\right)$ and $H_{0} \in H^{1}\left(\mathbb{R}^{3}, \mathbb{R}^{3}\right)$ satisfy the condition $\nabla \cdot\left(H_{0}+\beta \bar{u}_{0}\right)=0$ in $\mathscr{D}^{\prime}\left(\mathbb{R}^{3}\right)$. Then there exists a global weak solution $\left(u^{\varepsilon}, H^{\varepsilon}\right)$ to (1-19)+(1-17) under the initial-boundary conditions (1-8)-(1-10) such 
that for any $0<T<+\infty$,

$$
\begin{aligned}
\alpha_{1} \int_{0}^{T} \int_{\Omega}\left|\frac{\partial u^{\varepsilon}}{\partial t}\right|^{2}+\int_{0}^{T} \int_{\mathbb{R}^{3}} & \left(\left|\frac{\partial H^{\varepsilon}}{\partial t}\right|^{2}+\left|\nabla H^{\varepsilon}\right|^{2}\right) \\
+\left(\int_{\Omega} e_{\varepsilon}\left(u^{\varepsilon}\right)+\right. & \left.\int_{\mathbb{R}^{3}}\left(\frac{1}{2} \sigma\left|H^{\varepsilon}\right|^{2}+\left|\nabla H^{\varepsilon}\right|^{2}\right)\right)(T) \\
& \leq \int_{\mathbb{R}^{3}}\left|\nabla H_{0}\right|^{2}+e^{C T}\left(\int_{\Omega}\left|\nabla u_{0}\right|^{2}+\int_{\mathbb{R}^{3}}\left|H_{0}\right|^{2}\right),
\end{aligned}
$$

for some $C=C\left(\beta, \alpha_{1}\right)>0$.

Proof. For $N \geq 1$, let $\left(u_{N}, H_{N}\right) \in V_{N} \times W_{N}$ be given by (2-2) such that $u_{N}$ solves (2-3) and $H_{N}$ solves

$$
\int_{\mathbb{R}^{3}}\left(\nabla \times H_{N}\right) \cdot(\nabla \times \Psi)=-\sigma \int_{\mathbb{R}^{3}} \frac{\partial}{\partial t}\left(H_{N}+\beta \bar{u}_{N}\right) \cdot \Psi \quad \text { for all } \Psi \in W_{N}
$$

subject to the initial condition $\left.\left(u_{N}, H_{N}\right)\right|_{t=0}=\left(\Pi_{V_{N}}\left(u_{0}\right), \Pi_{W_{N}}\left(H_{0}\right)\right)$.

Testing (2-18) with $\Psi=H_{N}$ and integrating over $\mathbb{R}^{3}$ gives

$$
\text { 9) } \frac{d}{d t} \int_{\mathbb{R}^{3}} \frac{\sigma}{2}\left|H_{N}\right|^{2}+\int_{\mathbb{R}^{3}}\left|\nabla \times H_{N}\right|^{2}=-\beta \sigma \int_{\Omega} H_{N} \cdot \frac{\partial u_{N}}{\partial t} \leq \beta \sigma \int_{\Omega}\left|H_{N}\right|\left|\frac{\partial u_{N}}{\partial t}\right| \text {. }
$$

Combining (2-19) with (2-10) and applying the Cauchy-Schwarz inequality yields

$$
\begin{aligned}
\int_{\Omega} \alpha_{1}\left|\frac{\partial u_{N}}{\partial t}\right|^{2}+\frac{d}{d t}\left(\int_{\Omega} e_{\varepsilon}\left(u_{N}\right)+\int_{\mathbb{R}^{3}} \frac{1}{2} \sigma\left|H_{N}\right|^{2}\right) & +\int_{\mathbb{R}^{3}}\left|\nabla \times H_{N}\right|^{2} \\
& \leq C\left(\alpha_{1}, \beta\right) \int_{\mathbb{R}^{3}}\left|H_{N}\right|^{2} .
\end{aligned}
$$

This, combined with Grönwall's inequality, yields that for any $0<T<+\infty$,

$$
\begin{aligned}
\alpha_{1} \int_{0}^{T} \int_{\Omega}\left|\frac{\partial u_{N}}{\partial t}\right|^{2}+\int_{0}^{T} \int_{\mathbb{R}^{3}} \mid \nabla & \times\left. H_{N}\right|^{2}+\left(\int_{\Omega} e_{\varepsilon}\left(u_{N}\right)+\int_{\mathbb{R}^{3}} \frac{1}{2} \sigma\left|H_{N}\right|^{2}\right)(T) \\
& \leq e^{C T}\left(o(1)+\int_{\Omega}\left|\nabla u_{0}\right|^{2}+\int_{\mathbb{R}^{3}} \frac{1}{2} \sigma\left|H_{0}\right|^{2}\right)
\end{aligned}
$$

for some $C=C\left(\beta, \alpha_{1}\right)>0$, where we have used (2-7).

Now testing (2-18) with $\Psi=\partial H_{N} / \partial t$ and integrating over $\mathbb{R}^{3}$, we have

$$
\frac{d}{d t} \int_{\mathbb{R}^{3}} \frac{1}{2}\left|\nabla \times H_{N}\right|^{2}+\sigma \int_{\mathbb{R}^{3}}\left|\frac{\partial H_{N}}{\partial t}\right|^{2}=-\beta \sigma \int_{\Omega} \frac{\partial H_{N}}{\partial t} \cdot \frac{\partial u_{N}}{\partial t} .
$$

Thus, by the Cauchy-Schwarz inequality,

$$
\frac{d}{d t} \int_{\mathbb{R}^{3}}\left|\nabla \times H_{N}\right|^{2}+\sigma \int_{\mathbb{R}^{3}}\left|\frac{\partial H_{N}}{\partial t}\right|^{2} \leq 16 \beta^{2} \sigma \int_{\mathbb{R}^{3}}\left|\frac{\partial u_{N}}{\partial t}\right|^{2} .
$$


Integrating for $0 \leq t \leq T$ and applying (2-21), this implies

$$
\begin{aligned}
\int_{\mathbb{R}^{3}}\left|\nabla \times H_{N}\right|^{2}(T) & +\sigma \int_{0}^{T} \int_{\mathbb{R}^{3}}\left|\frac{\partial H_{N}}{\partial t}\right|^{2} \\
& \leq \int_{\mathbb{R}^{3}}\left|\nabla H_{0}\right|^{2}+16 \beta^{2} \sigma \int_{0}^{T} \int_{\Omega}\left|\frac{\partial u_{N}}{\partial t}\right|^{2} \\
& \leq \int_{\mathbb{R}^{3}}\left|\nabla H_{0}\right|^{2}+e^{C T}\left(o(1)+\int_{\Omega}\left|\nabla u_{0}\right|^{2}+\int_{\mathbb{R}^{3}}\left|H_{0}\right|^{2}\right) .
\end{aligned}
$$

Adding (2-21) and (2-24) together, we obtain

$$
\begin{aligned}
\alpha_{1} \int_{0}^{T} \int_{\Omega}\left|\frac{\partial u_{N}}{\partial t}\right|^{2} & +\int_{0}^{T} \int_{\mathbb{R}^{3}}\left(\left|\frac{\partial H_{N}}{\partial t}\right|^{2}+\left|\nabla \times H_{N}\right|^{2}\right) \\
+ & \left(\int_{\Omega} e_{\varepsilon}\left(u_{N}\right)+\int_{\mathbb{R}^{3}}\left(\frac{1}{2} \sigma\left|H_{N}\right|^{2}+\left|\nabla \times H_{N}\right|^{2}\right)\right)(T) \\
& \leq \int_{\mathbb{R}^{3}}\left|\nabla H_{0}\right|^{2}+e^{C T}\left(o(1)+\int_{\Omega}\left|\nabla u_{0}\right|^{2}+\int_{\mathbb{R}^{3}} \frac{1}{2} \sigma\left|H_{0}\right|^{2}\right)
\end{aligned}
$$

From (2-25) we may assume, after taking subsequences, that for any $0<T<+\infty$,

$$
\begin{gathered}
u_{N} \rightarrow u^{\varepsilon} \text { weak }^{*} \text { in } L^{\infty}\left([0, T], H^{1}(\Omega)\right), \quad \frac{\partial u_{N}}{\partial t} \rightarrow \frac{\partial u^{\varepsilon}}{\partial t} \text { in } L^{2}(\Omega \times[0, T]), \\
H_{N} \rightarrow H^{\varepsilon}, \frac{\partial H_{N}}{\partial t} \rightarrow \frac{\partial H^{\varepsilon}}{\partial t}, \nabla \times H_{N} \rightarrow \nabla \times H^{\varepsilon} \text { in } L^{2}\left(\mathbb{R}^{3} \times[0, T]\right) .
\end{gathered}
$$

As in Lemma 2.2, we can show that $\left(u^{\varepsilon}, H^{\varepsilon}\right)$ are weak solutions to (1-19)+(1-17), under the initial condition (1-8)-(1-10). By lower semicontinuity, we also see that (2-25) holds with $\left(u_{N}, H_{N}\right)$ replaced by $\left(u^{\varepsilon}, H^{\varepsilon}\right)$. To obtain the $L^{2}$-norm bound for $\nabla H$, we need to use the condition $\nabla \cdot\left(H_{0}+\beta \bar{u}_{0}\right)=0$ in $\mathscr{D}^{\prime}\left(\mathbb{R}^{3}\right)$. Note that

$$
\int_{\mathbb{R}^{3}}\left(\nabla \times H^{\varepsilon}\right) \cdot(\nabla \times \Psi)=-\sigma \int_{\mathbb{R}^{3}} \frac{\partial}{\partial t}\left(H^{\varepsilon}+\beta \bar{u}^{\varepsilon}\right) \cdot \Psi, \quad \forall \Psi \in H^{1}\left(\mathbb{R}^{3}\right) .
$$

Since $\delta>0$, by choosing $\Psi=\nabla \psi$ for $\psi \in C_{0}^{\infty}\left(\mathbb{R}^{3}\right)$ and observing $\nabla \times(\nabla \psi)=0$ in $\mathbb{R}^{3}$, we have

$$
\int_{\mathbb{R}^{3}} \frac{\partial}{\partial t}\left(H^{\varepsilon}+\beta \bar{u}^{\varepsilon}\right) \cdot \nabla \psi=0
$$

so that for a.e. $t>0$,

Thus

$$
\int_{\mathbb{R}^{3}} \nabla \cdot\left(H^{\varepsilon}+\beta \bar{u}^{\varepsilon}\right) \psi=\int_{\mathbb{R}^{3}} \nabla \cdot\left(H_{0}+\beta \bar{u}_{0}\right) \psi=0 .
$$

$$
\nabla \cdot\left(H^{\varepsilon}+\beta \bar{u}^{\varepsilon}\right)=0 \text { in } \mathscr{D}^{\prime}\left(\mathbb{R}^{3}\right) \text { for a.e. } t>0 .
$$


To proceed, we claim that

$$
\int_{\mathbb{R}^{3}}|\nabla H|^{2}=\int_{\mathbb{R}^{3}}\left(|\nabla \times H|^{2}+|\nabla \cdot H|^{2}\right) \quad \text { for all } H \in H^{1}\left(\mathbb{R}^{3}, \mathbb{R}^{3}\right) .
$$

Since $C_{0}^{\infty}\left(\mathbb{R}^{3}, \mathbb{R}^{3}\right)$ is dense in $H^{1}\left(\mathbb{R}^{3}, \mathbb{R}^{3}\right)$, it suffices to verify this inequality for $H \in C_{0}^{\infty}\left(\mathbb{R}^{3}, \mathbb{R}^{3}\right)$. This can be seen as follows:

$$
\begin{aligned}
\int_{\mathbb{R}^{3}}|\nabla H|^{2}= & \int_{\mathbb{R}^{3}} \sum_{i, j=1}^{3}\left(H_{j}^{i}\right)^{2}=\int_{\mathbb{R}^{3}} \sum_{i \neq j}\left(H_{i}^{j}\right)^{2}+\int_{\mathbb{R}^{3}} \sum_{i=1}^{3}\left(H_{i}^{i}\right)^{2} \\
= & \int_{\mathbb{R}^{3}}\left(\sum_{1 \leq i<j \leq 3}\left(H_{i}^{j}-H_{j}^{i}\right)^{2}+2 \sum_{1 \leq i<j \leq 3} H_{i}^{j} H_{j}^{i}\right) \\
& +\int_{\mathbb{R}^{3}}\left(\left(\sum_{i=1}^{3} H_{i}^{i}\right)^{2}-2 \sum_{1 \leq i<j \leq 3} H_{i}^{i} H_{j}^{j}\right) \\
= & \int_{\mathbb{R}^{3}}\left(|\nabla \times H|^{2}+|\nabla \cdot H|^{2}\right)+2 \int_{\mathbb{R}^{3}} \sum_{1 \leq i<j \leq 3}\left(H_{i}^{j} H_{j}^{i}-H_{i}^{i} H_{j}^{j}\right) \\
= & \int_{\mathbb{R}^{3}}\left(|\nabla \times H|^{2}+|\nabla \cdot H|^{2}\right),
\end{aligned}
$$

where the vanishing of $\int_{\mathbb{R}^{3}} \sum_{1 \leq i<j \leq 3}\left(H_{i}^{j} H_{j}^{i}-H_{i}^{i} H_{j}^{j}\right)=0$ in the last step comes from integrating by parts twice. Thus

$$
\int_{\mathbb{R}^{3}}\left|\nabla H^{\varepsilon}\right|^{2}=\int_{\mathbb{R}^{3}}\left(\left|\nabla \times H^{\varepsilon}\right|^{2}+\left|\nabla \cdot H^{\varepsilon}\right|^{2}\right) \leq C(\beta)\left(\int_{\mathbb{R}^{3}}\left|\nabla \times H^{\varepsilon}\right|^{2}+\int_{\Omega}\left|\nabla u^{\varepsilon}\right|^{2}\right)
$$

and hence (2-25), with $\left(u_{N}, H_{N}\right)=\left(u^{\varepsilon}, H^{\varepsilon}\right)$, yields (2-17).

For the system (1-19)+(1-18), we have:

Lemma 2.4. For any $u_{0} \in H^{1}\left(\Omega, S^{2}\right), H_{0} \in H^{1}\left(\mathbb{R}^{3}, \mathbb{R}^{3}\right)$ and $E_{0} \in H^{1}\left(\mathbb{R}^{3}, \mathbb{R}^{3}\right)$ with $\nabla \cdot E_{0}=\nabla \cdot H_{0}=0$ in $\mathscr{D}^{\prime}\left(\mathbb{R}^{3}\right)$, there exists a global weak solution $\left(u^{\varepsilon}, H^{\varepsilon}, E^{\varepsilon}\right)$ to (1-19)+(1-18) under the initial-boundary conditions (1-8)-(1-11) such that, for any $0<T<+\infty$,

$$
\begin{aligned}
& \int_{0}^{T} \int_{\Omega}\left|\frac{\partial u^{\varepsilon}}{\partial t}\right|^{2}+\mathscr{E}_{\varepsilon}(T) \\
& +\int_{\mathbb{R}^{3}}\left(\left|H^{\varepsilon}\right|^{2}+\left|E^{\varepsilon}\right|^{2}+\left|\frac{\partial H^{\varepsilon}}{\partial t}\right|^{2}+\left|\frac{\partial E^{\varepsilon}}{\partial t}\right|^{2}+\left|\nabla H^{\varepsilon}\right|^{2}+\left|\nabla E^{\varepsilon}\right|^{2}\right)(T) \\
& \quad \leq C\left(\varepsilon_{0}, \sigma, T\right)\left(\int_{\Omega}\left|\nabla u_{0}\right|^{2}+\int_{\mathbb{R}^{3}}\left(\left|H_{0}\right|^{2}+\left|E_{0}\right|^{2}+\left|\nabla H_{0}\right|^{2}+\left|\nabla E_{0}\right|^{2}\right)\right) .
\end{aligned}
$$

Proof. For $N \geq 1$, let $\left(u_{N}, H_{N}, E_{N}\right) \in V_{N} \times W_{N} \times W_{N}$ of the form (2-2) be a solution to (2-3)-(2-6). Since $\beta=0$ in this case, testing (2-4) with $\Psi=E_{N}$ and 
(2-5) with $\Psi=H_{N}$ and adding the resulting identities together gives

$$
\frac{d}{d t} \int_{\mathbb{R}^{3}}\left(\left|H_{N}\right|^{2}+\varepsilon_{0}\left|E_{N}\right|^{2}\right)+2 \sigma \int_{\mathbb{R}^{3}}\left|E_{N}\right|^{2}=0 .
$$

Differentiating (2-4) and (2-5) with respect to $t$ and testing the resulting equations with $\Psi=\partial E_{N} / \partial t$ and $\Psi=\partial H_{N} / \partial t$ respectively, we have

$$
\begin{aligned}
\int_{\mathbb{R}^{3}}\left(\frac{\partial E_{N}}{\partial t} \cdot \nabla \times \frac{\partial H_{N}}{\partial t}-\frac{\partial H_{N}}{\partial t} \cdot \nabla \times \frac{\partial E_{N}}{\partial t}\right) \\
=\int_{\mathbb{R}^{3}}\left(\frac{\partial E_{N}}{\partial t} \cdot\left(\varepsilon_{0} \frac{\partial^{2} E_{N}}{\partial t^{2}}+\sigma \frac{\partial E_{N}}{\partial t}\right)+\frac{\partial H_{N}}{\partial t} \cdot \frac{\partial^{2} H_{N}}{\partial t^{2}}\right) .
\end{aligned}
$$

Since

$$
\int_{\mathbb{R}^{3}}\left(\frac{\partial E_{N}}{\partial t} \cdot \nabla \times \frac{\partial H_{N}}{\partial t}-\frac{\partial H_{N}}{\partial t} \cdot \nabla \times \frac{\partial E_{N}}{\partial t}\right)=0
$$

we obtain

$$
\frac{d}{d t} \int_{\mathbb{R}^{3}}\left(\varepsilon_{0}\left|\frac{\partial E_{N}}{\partial t}\right|^{2}+\left|\frac{\partial H_{N}}{\partial t}\right|^{2}\right)+2 \sigma \int_{\mathbb{R}^{3}}\left|\frac{\partial E_{N}}{\partial t}\right|^{2}=0 .
$$

Combining (2-27) with (2-28), we get

$$
\begin{aligned}
\frac{d}{d t} \int_{\mathbb{R}^{3}}\left(\left|H_{N}\right|^{2}+\varepsilon_{0}\left(\left|E_{N}\right|^{2}+\left|\frac{\partial E_{N}}{\partial t}\right|^{2}\right)\right. & \left.+\left|\frac{\partial H_{N}}{\partial t}\right|^{2}\right) \\
& =-2 \sigma \int_{\mathbb{R}^{3}}\left(\left|E_{N}\right|^{2}+\left|\frac{\partial E_{N}}{\partial t}\right|^{2}\right) .
\end{aligned}
$$

Since

$$
\left.\frac{\partial H_{N}}{\partial t}\right|_{t=0}=-\nabla \times\left(\Pi_{W_{N}}\left(E_{0}\right)\right),\left.\quad \varepsilon_{0} \frac{\partial E}{\partial t}\right|_{t=0}=\nabla \times\left(\Pi_{W_{N}}\left(H_{0}\right)\right)-\sigma \Pi_{W_{N}}\left(E_{0}\right),
$$

integrating (2-29) for $0 \leq t \leq T$ yields

$$
\begin{gathered}
\int_{\mathbb{R}^{3}}\left(\left|H_{N}\right|^{2}+\varepsilon_{0}\left(\left|E_{N}\right|^{2}+\left|\frac{\partial E_{N}}{\partial t}\right|^{2}\right)+\left|\frac{\partial H_{N}}{\partial t}\right|^{2}\right)(T) \\
\quad+2 \sigma \int_{0}^{T} \int_{\mathbb{R}^{3}}\left(\left|E_{N}\right|^{2}+\left|\frac{\partial E_{N}}{\partial t}\right|^{2}\right) \\
\leq \int_{\mathbb{R}^{3}}\left(\left|\Pi_{W_{N}}\left(H_{0}\right)\right|^{2}+\varepsilon_{0}\left|\Pi_{W_{N}}\left(E_{0}\right)\right|^{2}+\left|\nabla \times\left(\Pi_{W_{N}}\left(E_{0}\right)\right)\right|^{2}\right. \\
\left.+\varepsilon_{0}^{-1}\left|\nabla \times\left(\Pi_{W_{N}}\left(H_{0}\right)\right)-\sigma \Pi_{W_{N}}\left(E_{0}\right)\right|^{2}\right) \\
\leq C\left(\varepsilon_{0}, \sigma\right) \int_{\mathbb{R}^{3}}\left(\left|H_{0}\right|^{2}+\left|E_{0}\right|^{2}+\left|\nabla H_{0}\right|^{2}+\left|\nabla E_{0}\right|^{2}\right) .
\end{gathered}
$$


For $u_{N}$, by testing (2-3) with $\Phi=\partial u_{N} / \partial t$ as in (2-10) of Lemma 2.2, we have

$$
\alpha_{1} \int_{\Omega}\left|\frac{\partial u_{N}}{\partial t}\right|^{2}+\frac{d}{d t} \int_{\Omega} e_{\varepsilon}\left(u_{N}\right) \leq C \int_{\mathbb{R}^{3}}\left|H_{N}\right|^{2} .
$$

This, with the help of (2-30) and (2-7), implies that for any $0<T<+\infty$,

$$
\begin{aligned}
& \alpha_{1} \int_{0}^{T} \int_{\Omega}\left|\frac{\partial u_{N}}{\partial t}\right|^{2}+\int_{\Omega} e_{\varepsilon}\left(u_{N}(T)\right) \\
& \quad \leq C T \int_{\mathbb{R}^{3}}\left(\left|H_{0}\right|^{2}+\left|E_{0}\right|^{2}+\left|\nabla H_{0}\right|^{2}+\left|\nabla E_{0}\right|^{2}\right)+\int_{\Omega}\left|\nabla u_{0}\right|^{2}+o(1)
\end{aligned}
$$

It follows from (2-30), (2-4), and (2-5) with $\beta=0$ that

$$
\begin{aligned}
\int_{\mathbb{R}^{3}}\left(\left|\nabla \times H_{N}\right|^{2}+\left|\nabla \times E_{N}\right|^{2}\right)(T) & \leq C \int_{\mathbb{R}^{3}}\left(\left|\frac{\partial E_{N}}{\partial t}\right|^{2}+\left|E_{N}\right|^{2}+\left|\frac{\partial H_{N}}{\partial t}\right|^{2}\right)(T) \\
& \leq C \int_{\mathbb{R}^{3}}\left(\left|H_{0}\right|^{2}+\left|E_{0}\right|^{2}+\left|\nabla H_{0}\right|^{2}+\left|\nabla E_{0}\right|^{2}\right) .
\end{aligned}
$$

From (2-30), (2-32), and (2-33) we may assume, after taking subsequences, that for any $0<T<+\infty$,

$$
\begin{gathered}
u_{N} \rightarrow u^{\varepsilon} \text { weak }^{*} \text { in } L^{\infty}\left([0, T], H^{1}(\Omega)\right), \quad \frac{\partial u_{N}}{\partial t} \rightarrow \frac{\partial u^{\varepsilon}}{\partial t} \text { in } L^{2}(\Omega \times[0, T]), \\
H_{N} \rightarrow H^{\varepsilon}, \frac{\partial H_{N}}{\partial t} \rightarrow \frac{\partial H^{\varepsilon}}{\partial t}, \nabla \times H_{N} \rightarrow \nabla \times H^{\varepsilon} \text { in } L^{2}\left(\mathbb{R}^{3} \times[0, T]\right), \\
E_{N} \rightarrow E^{\varepsilon}, \frac{\partial E_{N}}{\partial t} \rightarrow \frac{\partial E^{\varepsilon}}{\partial t}, \nabla \times E_{N} \rightarrow \nabla \times E^{\varepsilon} \text { in } L^{2}\left(\mathbb{R}^{3} \times[0, T]\right) .
\end{gathered}
$$

As in the previous lemmas, it is a standard exercise to check that $\left(u^{\varepsilon}, H^{\varepsilon}, E^{\varepsilon}\right)$ solves (1-19)+(1-18) and the initial-boundary conditions (1-8)-(1-11). Moreover, by lower semicontinuity, we have, for $0<T<+\infty$,

$$
\begin{aligned}
& \int_{0}^{T} \int_{\Omega}\left|\frac{\partial u^{\varepsilon}}{\partial t}\right|^{2}+\mathscr{E}_{\varepsilon}(T) \\
& +\int_{\mathbb{R}^{3}}\left(\left|H^{\varepsilon}\right|^{2}+\left|E^{\varepsilon}\right|^{2}+\left|\frac{\partial H^{\varepsilon}}{\partial t}\right|^{2}+\left|\frac{\partial E^{\varepsilon}}{\partial t}\right|^{2}+\left|\nabla \times H^{\varepsilon}\right|^{2}+\left|\nabla \times E^{\varepsilon}\right|^{2}\right) \\
& \quad \leq C\left(\varepsilon_{0}, \sigma, T\right)\left(\int_{\Omega}\left|\nabla u_{0}\right|^{2}+\int_{\mathbb{R}^{3}}\left(\left|H_{0}\right|^{2}+\left|E_{0}\right|^{2}+\left|\nabla H_{0}\right|^{2}+\left|\nabla E_{0}\right|^{2}\right)\right) .
\end{aligned}
$$

As in the previous lemma, we can check that $\nabla \cdot H_{0}=\nabla \cdot E_{0}=0$ is preserved under (1-18), that is,

$$
\nabla \cdot H^{\varepsilon}(t)=\nabla \cdot E^{\varepsilon}(t)=0 \text { a.e. } t>0 .
$$

Finally, it is not hard to see that (2-34) and (2-35) yield (2-26). 
Remark 2.5. Lemmas 2.3 and 2.4 show that, for any $0<T<+\infty, H^{\varepsilon}$ is uniformly bounded in $L^{\infty}\left([0, T], H^{1}\left(\mathbb{R}^{3}\right)\right)$. Hence by the Sobolev embedding inequality, $H^{\varepsilon}$ is uniformly bounded in $L^{\infty}\left([0, T], L^{6}\left(\mathbb{R}^{3}\right)\right)$. This property plays an important role in the proof of $C^{\alpha}$-regularity of $\nabla u$ claimed in Theorems 1.3 and 1.4.

We end this section with a local energy inequality.

Lemma 2.6. There exists $C>0$ such that for any $\varepsilon>0, u_{0} \in H^{1}\left(\Omega, S^{2}\right), H_{0} \in$ $L^{2}\left(\mathbb{R}^{3}, \mathbb{R}^{3}\right)$ and $E_{0} \in L^{2}\left(\mathbb{R}^{3}, \mathbb{R}^{3}\right)$, if $\left(u^{\varepsilon}, H^{\varepsilon}, E^{\varepsilon}\right)$ is the global weak solution of $(1-19)+(1-2)-(1-3)$ with conditions (1-8)+(1-9)-(1-11) obtained in Lemma 2.2, then for any $x_{0} \in \Omega, t_{0}>0$, and $0<r<\min \left\{\operatorname{dist}\left(x_{0}, \partial \Omega\right), \sqrt{t}_{0} / 2\right\}$, we have

$$
\begin{aligned}
r^{-1} \int_{P_{r / 2}\left(z_{0}\right)}\left|\frac{\partial u^{\varepsilon}}{\partial t}\right|^{2}+r^{-1} \max _{t \in\left[t_{0}-r^{2} / 4, t_{0}\right]} \int_{B_{r / 2}\left(x_{0}\right)} e_{\varepsilon}\left(u^{\varepsilon}\right) \\
\quad \leq C r^{-3} \int_{P_{r}\left(z_{0}\right)} e_{\varepsilon}\left(u^{\varepsilon}\right)+C r^{-1} \int_{P_{r}\left(z_{0}\right)}\left|H^{\varepsilon}\right|^{2}
\end{aligned}
$$

Proof. Write $(u, H)$ for $\left(u^{\varepsilon}, H^{\varepsilon}\right)$. For $x_{0} \in \Omega$ and $0<r<\min \left\{\operatorname{dist}\left(x_{0}, \partial \Omega\right), \sqrt{t_{0}}\right\}$, by Fubini's theorem there is $\alpha \in\left(\frac{1}{2}, \frac{7}{8}\right)$ such that

$$
\int_{B_{r}\left(x_{0}\right)} e_{\varepsilon}(u)\left(t_{0}-\alpha^{2} r^{2}\right) \leq 8 r^{-2} \int_{P_{r}\left(z_{0}\right)} e_{\varepsilon}(u) .
$$

Let $\phi(x) \in C_{0}^{\infty}\left(B_{r}\left(x_{0}\right)\right)$ be such that $0 \leq \phi \leq 1, \phi \equiv 1$ on $B_{r / 2}\left(x_{0}\right)$. Multiplying (1-19) by $\phi^{2}(\partial u / \partial t)$ and integrating over $B_{r}\left(x_{0}\right)$, we get

$$
\begin{aligned}
& \alpha_{1} \int_{B_{r}\left(x_{0}\right)}\left|\frac{\partial u}{\partial t}\right|^{2} \phi^{2}+\frac{d}{d t} \int_{B_{r}\left(x_{0}\right)} e_{\varepsilon}(u) \phi^{2} \\
& \quad=-2 \int_{B_{r}\left(x_{0}\right)} \phi \nabla \phi \nabla u \cdot \frac{\partial u}{\partial t}-\int_{B_{r}\left(x_{0}\right)} \phi^{2} u \times(H \times u) \cdot \frac{\partial u}{\partial t} \\
& \leq \frac{\alpha_{1}}{2} \int_{B_{r}\left(x_{0}\right)}\left|\frac{\partial u}{\partial t}\right|^{2} \phi^{2}+C\left(\alpha_{1}\right) \int_{B_{r}\left(x_{0}\right)}\left(|\nabla \phi|^{2}|\nabla u|^{2}+\phi^{2}|H|^{2}\right)
\end{aligned}
$$

Integrating (2-38) from $t_{0}-\alpha^{2} r^{2}$ to $t \in\left[t_{0}-r^{2} / 4, t_{0}\right]$ and applying (2-37), we obtain (2-36).

\section{Energy monotonicity on time slices}

An energy monotonicity property analogous to that of [Struwe 1988] (see also [Chen and Struwe 1989; Chen and Lin 1993]) is unknown for Landau-Lifshitz type equations. In order to derive an prior estimate for $\left(u^{\varepsilon}, E^{\varepsilon}, H^{\varepsilon}\right)$ under the small energy condition, we need an energy monotonicity of $u^{\varepsilon}$ on time slices, which can be derived by a Pohozaev-type argument as in [Wang 2006]. 
Lemma 3.1. For $\varepsilon>0$, let $\left(u^{\varepsilon}, H^{\varepsilon}\right)$ be a weak solution to (1-19). For a.e. $t>0$, any $x_{0} \in \Omega$, and $0<r \leq R<\min \left\{1\right.$, $\left.\operatorname{dist}\left(x_{0}, \partial \Omega\right)\right\}$, we have

$$
\begin{gathered}
r^{-1} E_{\varepsilon}\left(u^{\varepsilon}, B_{r}\left(x_{0}\right)\right) \leq 2 R^{-1} E_{\varepsilon}\left(u^{\varepsilon}, B_{R}\left(x_{0}\right)\right)+C_{0} R \int_{B_{R}\left(x_{0}\right)}\left(\left|\frac{\partial u^{\varepsilon}}{\partial t}\right|^{2}+\left|H^{\varepsilon}\right|^{2}\right) \\
\int_{B_{R}}\left|x-x_{0}\right|^{-1} \frac{\left(1-\left|u^{\varepsilon}\right|^{2}\right)^{2}}{\varepsilon^{2}} \\
\leq 2 R^{-1} E_{\varepsilon}\left(u^{\varepsilon}, B_{R}\left(x_{0}\right)\right)+C_{0} R \int_{B_{R}\left(x_{0}\right)}\left(\left|\frac{\partial u^{\varepsilon}}{\partial t}\right|^{2}+\left|H^{\varepsilon}\right|^{2}\right)
\end{gathered}
$$

for some $C_{0}=C_{0}\left(\alpha_{1}\right)>0$, where

$$
E_{\varepsilon}\left(u^{\varepsilon}, A\right)=\int_{A}\left(\frac{1}{2}\left|\nabla u^{\varepsilon}\right|^{2}+\frac{\left(1-\left|u^{\varepsilon}\right|^{2}\right)^{2}}{2 \varepsilon^{2}}\right), \quad A \subseteq \mathbb{R}^{3} .
$$

Proof. The proof is a modification of [Wang 2006] (see also [Ding and Guo 2004; Melcher 2005]). We sketch it here. First observe that for a.e. $t>0$, we have $\Delta u \in L^{2}(\Omega)$ and hence $\nabla^{2} u \in L^{2}(\Omega)$. For $p \in \mathbb{R}^{3}$, define $R(p): \mathbb{R}^{3} \rightarrow \mathbb{R}^{3}$ by

$$
R(p)(v)=\alpha_{1} v-\alpha_{2} p \times v \quad \text { for all } v \in \mathbb{R}^{3} .
$$

Assume $x_{0}=0 \in \Omega$. Write $(u, H)=\left(u^{\varepsilon}, H^{\varepsilon}\right)$ and $B_{r}=B_{r}(0)$. Multiplying (1-19) by $x \cdot \nabla u$ and integrating over $B_{r}$ yields

$$
\begin{aligned}
& \int_{B_{r}}\left\langle R(u)\left(\frac{\partial u}{\partial t}\right)+u \times(u \times H), x \cdot \nabla u\right\rangle \\
& \quad=\int_{B_{r}}\left\langle\Delta u+\frac{1}{\varepsilon^{2}}\left(1-|u|^{2}\right) u, x \cdot \nabla u\right\rangle \\
& \quad=r \int_{\partial B_{r}}\left(\left|\frac{\partial u}{\partial r}\right|^{2}-\frac{1}{2}|\nabla u|^{2}-\frac{\left(1-|u|^{2}\right)^{2}}{4 \varepsilon^{2}}\right)+\int_{B_{r}}\left(\frac{1}{2}|\nabla u|^{2}+\frac{3\left(1-|u|^{2}\right)^{2}}{4 \varepsilon^{2}}\right) \\
& \quad \geq r \int_{\partial B_{r}}\left(\left|\frac{\partial u}{\partial r}\right|^{2}-\frac{1}{2}|\nabla u|^{2}-\frac{\left(1-|u|^{2}\right)^{2}}{4 \varepsilon^{2}}\right)+E_{\varepsilon}\left(u, B_{r}\right) .
\end{aligned}
$$

Hence we have

$$
\begin{aligned}
& \frac{d}{d r}\left(r^{-1} E_{\varepsilon}\left(u, B_{r}\right)-r^{-1} \int_{B_{r}}\left\langle R(u)\left(\frac{\partial u}{\partial t}\right)+u \times(u \times H), x \cdot \nabla u\right\rangle\right) \\
& \geq r^{-1} \int_{\partial B_{r}}\left(\left|\frac{\partial u}{\partial r}\right|^{2}+\frac{\left(1-|u|^{2}\right)^{2}}{4 \varepsilon^{2}}\right)-r^{-1} \int_{\partial B_{r}}\left\langle R(u) \frac{\partial u}{\partial t}+u \times(u \times H), x \cdot \nabla u\right\rangle .
\end{aligned}
$$

Since $|u| \leq 1$, we have $|u \times(u \times H)| \leq|H|$ and $|R(u)(\partial u / \partial t)| \leq|\partial u / \partial t|$. The second term of the right hand side of (3-4) can be estimated by 


$$
\begin{aligned}
-r^{-1} \int_{\partial B_{r}}\left\langle R(u)\left(\frac{\partial u}{\partial t}\right)+u\right. & \times(u \times H), x \cdot \nabla u \mid \\
& \geq-\frac{1}{4} r^{-1} \int_{\partial B_{r}}\left|\frac{\partial u}{\partial r}\right|^{2}-r \int_{\partial B_{r}}\left(\left|\frac{\partial u}{\partial t}\right|^{2}+|H|^{2}\right) .
\end{aligned}
$$

The second term of the left hand side of (3-4) can be estimated by

$$
\begin{aligned}
\mid r^{-1} \int_{B_{r}}\left\langle R(u)\left(\frac{\partial u}{\partial t}\right)+u \times(u \times H)\right. & , x \cdot \nabla u|| \\
& \leq \frac{1}{4} E_{\varepsilon}\left(u, B_{r}\right)+r \int_{B_{r}}\left(\left|\frac{\partial u}{\partial t}\right|^{2}+|H|^{2}\right) .
\end{aligned}
$$

Putting (3-5) and (3-6) into (3-4) and integrating from $r$ to $R$ gives

$$
\begin{aligned}
& 2 R^{-1} E_{\varepsilon}\left(u, B_{R}\right)+R \int_{B_{R}}\left(\left|\frac{\partial u}{\partial t}\right|^{2}+|H|^{2}\right) \\
& \geq \frac{1}{2} r^{-1} E_{\varepsilon}\left(u, B_{r}\right)-r \int_{B_{r}}\left(\left|\frac{\partial u}{\partial t}\right|^{2}+|H|^{2}\right) \\
& \quad+\int_{B_{R} \backslash B_{r}} \frac{1}{|x|}\left(\left|\frac{\partial u}{\partial r}\right|^{2}+\frac{\left(1-|u|^{2}\right)^{2}}{4 \varepsilon^{2}}\right)-\int_{0}^{R} s \int_{\partial B_{s}}\left(\left|\frac{\partial u}{\partial t}\right|^{2}+|H|^{2}\right)
\end{aligned}
$$

Since

and

$$
r \int_{B_{r}}\left(\left|\frac{\partial u}{\partial t}\right|^{2}+|H|^{2}\right) \leq R \int_{B_{R}}\left(\left|\frac{\partial u}{\partial t}\right|^{2}+|H|^{2}\right)
$$

$$
\int_{0}^{R} s \int_{\partial B_{s}}\left(\left|\frac{\partial u}{\partial t}\right|^{2}+|H|^{2}\right) \leq R \int_{\partial B_{R}}\left(\left|\frac{\partial u}{\partial t}\right|^{2}+|H|^{2}\right)
$$

(3-7) clearly implies both (3-1) and (3-2).

\section{On the lower bound of $\left|u^{\varepsilon}\right|$}

We will now establish a lower bound estimate of $\left|u^{\varepsilon}\right|$ on generic time slices, under the smallness condition $r^{-3} \int_{P_{r}} e_{\varepsilon}\left(u^{\varepsilon}\right)$.

Definition 4.1. For any $\varepsilon \in\left(0, \frac{1}{2}\right), x_{0} \in \Omega, t_{0}>0,0<r<\min \left\{\operatorname{dist}\left(x_{0}, \partial \Omega\right), \sqrt{t_{0}}\right\}$, and $\Lambda>0$, we define the set of good time slices by

$$
G_{z_{0}, r}^{\Lambda}=\left\{t \in\left[t_{0}-r^{2}, t_{0}\right): \int_{B_{r}\left(x_{0}\right)}\left|\frac{\partial u^{\varepsilon}}{\partial t}\right|^{2} \leq \frac{\Lambda^{2}}{r^{2}} \int_{P_{r}\left(z_{0}\right)}\left|\frac{\partial u^{\varepsilon}}{\partial t}\right|^{2}\right\}
$$

and the set of bad time slices by

$$
B_{z_{0}, r}^{\Lambda}=\left[t_{0}-r^{2}, t_{0}\right) \backslash G_{z_{0}, r}^{\Lambda}
$$


By Fubini's theorem,

$$
\left|B_{z_{0}, r}^{\Lambda}\right| \leq \frac{r^{2}}{\Lambda^{2}} .
$$

Similarly to [Melcher 2005; Wang 2006], we have:

Lemma 4.2. For $\varepsilon>0$, let $\left(u^{\varepsilon}, H^{\varepsilon}\right)$ be the weak solution of (1-19) in Lemma 2.2. Denote

$$
\left\|H^{\varepsilon}\right\|_{L_{t}^{\infty} L_{x}^{2}\left(\mathbb{R}^{3} \times\left[0, t_{0}\right]\right)}=C_{0} .
$$

Then for any $\Lambda>0$, there exist $\eta_{0}>0$ and $r_{0}>0$ depending on $\Lambda$ and $C_{0}$ such that for any $z_{0}=\left(x_{0}, t_{0}\right) \in \Omega \times(0,+\infty)$ and $0<r<\min \left\{\operatorname{dist}\left(x_{0}, \partial \Omega\right), \sqrt{t_{0}}, r_{0}\right\}$ if

$$
r^{-3} \int_{P_{r}\left(z_{0}\right)} e_{\varepsilon}\left(u^{\varepsilon}\right) \leq \eta_{0}^{2},
$$

then

$$
\left|u^{\varepsilon}\right|(x, t) \geq \frac{1}{2} \quad \text { for all } x \in B_{r / 4}\left(x_{0}\right) \text { and } t \in G_{z_{0}, r / 2}^{\Lambda} .
$$

Proof. The proof is a modification of [Melcher 2005; Wang 2006]. We prove a $C^{1 / 2}$-estimate of $u^{\varepsilon}(\cdot, s)$ for $s \in G_{z_{0}, r / 2}^{\Lambda}$ (see also [Melcher 2005, page 577, Lemma 5]). Define $v^{\varepsilon}(x, t)=u^{\varepsilon}\left(x_{0}+\varepsilon x, s+\varepsilon^{2} t\right): B_{2} \times[-4,4] \rightarrow \mathbb{R}^{3}$. Then $w^{\varepsilon}(x) \equiv v^{\varepsilon}(x, 0)$ satisfies

$$
\Delta w^{\varepsilon}=R\left(w^{\varepsilon}\right)\left(\frac{\partial v^{\varepsilon}}{\partial t}(0)\right)-\left(1-\left|w^{\varepsilon}\right|^{2}\right) w^{\varepsilon}-w^{\varepsilon} \times\left(\widetilde{H}^{\varepsilon} \times w^{\varepsilon}\right),
$$

where $\widetilde{H}^{\varepsilon}(x)=\varepsilon^{2} H^{\varepsilon}(\varepsilon x, s)$. By the standard $W^{2,2}$ estimate, we have

$$
\begin{aligned}
\left\|\nabla^{2} w^{\varepsilon}\right\|_{L^{2}\left(B_{1}\right)}^{2} & \leq C\left(1+\left\|\frac{\partial w^{\varepsilon}}{\partial t}\right\|_{L^{2}\left(B_{2}\right)}^{2}+\left\|\widetilde{H}^{\varepsilon}\right\|_{L^{2}\left(B_{2}\right)}^{2}\right) \\
& \leq C\left(1+\varepsilon \int_{B_{2 \varepsilon}\left(x_{0}\right)}\left(\left|\frac{\partial u^{\varepsilon}}{\partial t}\right|^{2}+\left|H^{\varepsilon}\right|^{2}\right)(s)\right) \\
& \leq C\left(1+C_{0}^{2}+r \int_{B_{r / 2}\left(x_{0}\right)}\left|\frac{\partial u^{\varepsilon}}{\partial t}\right|^{2}(s)\right) \leq C\left(1+C_{0}^{2}+\Lambda^{2} \varepsilon_{0}^{2}\right),
\end{aligned}
$$

where we have used both (4-1) and Lemma 2.6 in the last step. Therefore, by the Sobolev embedding theorem, $w^{\varepsilon} \in C^{1 / 2}\left(B_{1}\right)$. Moreover, by rescaling and (4-7),

$$
\left(u^{\varepsilon}(s)\right)_{C^{1 / 2}\left(B_{r / 2}\left(x_{0}\right)\right)} \leq C\left(\Lambda, \eta_{0}, C_{0}\right) \varepsilon^{-1 / 2} \quad \text { for all } s \in G_{z_{0}, r / 2}^{\Lambda} .
$$

Suppose that (4-5) were false. Then there exists $z_{1}=\left(x_{1}, t_{1}\right) \in B_{r / 4}\left(x_{0}\right) \times G_{z_{0}, r / 2}^{\Lambda}$ such that $\left|u^{\varepsilon}\left(z_{1}\right)\right|<\frac{1}{2}$. Hence for sufficiently small $\theta_{0}>0$, if $y \in B_{\theta_{0}^{2} \varepsilon}\left(x_{1}\right)$, we would have

$$
\left|u^{\varepsilon}\right|\left(y, t_{1}\right) \leq\left|u^{\varepsilon}\right|\left(x_{1}, t_{1}\right)+\left(u^{\varepsilon}\left(t_{1}\right)\right)_{C^{1 / 2}}\left|y-x_{1}\right|^{1 / 2} \leq \frac{1}{2}+C\left(\Lambda, \eta_{0}, C_{0}\right) \theta_{0} \leq \frac{3}{4},
$$


so that

$$
\int_{B_{\theta_{0}^{2}}\left(x_{1}\right)}\left|x-x_{1}\right|^{-1} \frac{\left(1-\left|u^{\varepsilon}\right|^{2}\right)^{2}\left(x, t_{1}\right)}{\varepsilon^{2}} \geq C_{1} .
$$

At the same time, the bound (4-4) gives $\sup _{x \in B_{r / 2}\left(x_{0}\right)}\left(\frac{r}{2}\right)^{-3} \int_{P_{r / 2}\left(x, t_{0}\right)} e_{\varepsilon}\left(u^{\varepsilon}\right) \leq 8 \eta_{0}^{2}$. This,
combined with Lemma 2.6, implies

$$
\sup _{t \in\left[t_{0}-r^{2} / 16, t_{0}\right)} \sup _{x \in B_{r / 4}\left(x_{0}\right)}\left(\frac{r}{4}\right)^{-1} \int_{B_{r / 4}(x)} e_{\varepsilon}\left(u^{\varepsilon}\right) \leq C\left(\eta_{0}^{2}+C_{0}^{2} r\right) .
$$

By the definition of $G_{z_{0}, r / 2}^{\Lambda}$ and Lemma 2.6, we have

$$
\begin{aligned}
& \sup _{t \in G_{z_{0}, r / 2}^{\Lambda}} \sup _{x \in B_{r / 4}\left(x_{0}\right)} r \int_{B_{r / 4}(x)}\left|\frac{\partial u^{\varepsilon}}{\partial t}\right|^{2}(t) \\
& \leq \sup _{t \in G_{z_{0}, r / 2}^{\Lambda}} r \int_{B_{r / 2}\left(x_{0}\right)}\left|\frac{\partial u^{\varepsilon}}{\partial t}\right|^{2}(t) \\
& \leq C\left(\frac{\Lambda^{2}}{r^{3}} \int_{P_{r}\left(z_{0}\right)} e_{\varepsilon}\left(u^{\varepsilon}\right)+\Lambda^{2} r\left\|H^{\varepsilon}\right\|_{L_{t}^{\infty} L_{x}^{2}}^{2}\right) \leq C \Lambda^{2}\left(\eta_{0}^{2}+C_{0}^{2} r\right)
\end{aligned}
$$

With (4-10), (4-11), and the monotonicity inequality (3-2), we obtain

$$
\begin{aligned}
\int_{B_{\theta^{2} \varepsilon}\left(x_{1}\right)} & \left|x-x_{1}\right|^{-1} \frac{\left(1-\left|u^{\varepsilon}\right|^{2}\right)^{2}}{\varepsilon_{0}^{2}}\left(t_{1}\right) \\
& \leq C\left(r^{-1} \int_{B_{r / 4}\left(x_{1}\right)} e_{\varepsilon}\left(u^{\varepsilon}\right)\left(t_{1}\right)+r \int_{B_{r / 4}\left(x_{1}\right)}\left(\left|\frac{\partial u^{\varepsilon}}{\partial t}\right|^{2}+\left|H^{\varepsilon}\right|^{2}\right)\left(t_{1}\right)\right) \\
& \leq C\left(\Lambda^{2} \eta_{0}^{2}+C_{0}^{2} r_{0}\right) .
\end{aligned}
$$

This contradicts (4-9) provided $r_{0}>0$ and $\eta_{0}>0$ are chosen sufficiently small.

\section{Energy decay estimates and proof of Theorem 1.2}

In this section, we first establish the decay estimate of the normalized energy $r^{-3} \int_{P_{r}(z)} e_{\varepsilon}\left(u^{\varepsilon}\right)$, provided that it is sufficiently small. Then we give a proof of Theorem 1.2. The techniques employed in the proof are suitable modifications of that by Hélein [1990] and Evans [1991] in the context of harmonic maps.

Lemma 5.1. For any $L>0$ and $\delta>0$, there exist $C(\delta)>0, \eta(\delta)>0$, and $\varepsilon_{1}(\delta)>0$, such that if $\left(u^{\varepsilon}, H^{\varepsilon}\right)$ is the weak solution of (1-19) in Lemma 2.2 and we take $z_{0}=\left(x_{0}, t_{0}\right) \in \Omega \times \mathbb{R}^{+}, 0<r<\min \left\{\operatorname{dist}\left(x_{0}, \partial \Omega\right), \sqrt{t_{0}}, \varepsilon_{1}^{2}(\delta) / L^{2}\right\}$, and $0<\varepsilon \leq \eta(\delta) r$ satisfying

$$
\left\|H^{\varepsilon}\right\|_{L_{t}^{\infty} L_{x}^{2}\left(P_{r}\left(z_{0}\right)\right)} \leq L \quad \text { and } \quad r^{-3} \int_{P_{r}\left(z_{0}\right)} e_{\varepsilon}\left(u^{\varepsilon}\right) \leq \varepsilon_{1}^{2}(\delta),
$$


then we have

$$
\begin{aligned}
\left(\frac{r}{8}\right)^{-3} \int_{P_{r / 8}\left(z_{0}\right)} e_{\varepsilon}\left(u^{\varepsilon}\right) \leq \delta\left(r^{-3} \int_{P_{r}\left(z_{0}\right)} e_{\varepsilon}\left(u^{\varepsilon}\right)\right. & \left.+r\left\|H^{\varepsilon}\right\|_{L_{t}^{\infty} L_{x}^{2}\left(P_{r}\left(z_{0}\right)\right)}^{2}\right) \\
& +\frac{C(\delta)}{\delta} r^{-5} \int_{P_{r}\left(z_{0}\right)}\left|u^{\varepsilon}-u_{P_{r}\left(z_{0}\right)}^{\varepsilon}\right|^{2},
\end{aligned}
$$

where $u_{P_{r}\left(z_{0}\right)}^{\varepsilon}$ is the average of $u^{\varepsilon}$ over $P_{r}\left(z_{0}\right)$ :

$$
u_{P_{r}\left(z_{0}\right)}^{\varepsilon}=\frac{1}{\left|P_{r}\left(z_{0}\right)\right|} \int_{P_{r}\left(z_{0}\right)} u^{\varepsilon}, \quad r>0 .
$$

Proof. We follow [Wang 2006, page 1631, Proposition 5.1] with suitable modifications, and outline the key steps here. For simplicity, write $(u, H)=\left(u^{\varepsilon}, H^{\varepsilon}\right)$ and assume $z_{0}=\left(x_{0}, t_{0}\right)=(0,1) \in \Omega \times \mathbb{R}_{+}$. For $r>0$, let $u_{r}(x, t)=u\left(r x, 1+r^{2} t\right)$ and $H_{r}(x, t)=r^{2} H\left(r x, 1+r^{2} t\right)$ for $(x, t) \in P_{1}$. Then it follows from (1-19) that $\left(u_{r}, H_{r}\right)$ satisfies

$$
R\left(u_{r}\right)\left(\frac{\partial u_{r}}{\partial t}\right)=\Delta u_{r}+\frac{\left(1-\left|u_{r}\right|^{2}\right)}{\hat{\varepsilon}^{2}} u_{r}+u_{r} \times\left(H_{r} \times u_{r}\right) \quad \text { in } P_{1},
$$

where $\hat{\varepsilon}=r^{-1} \varepsilon$. Moreover,

$$
\begin{gathered}
\int_{P_{1}} e_{\hat{\varepsilon}}\left(u_{r}\right)=r^{-3} \int_{P_{r}(0,1)} e_{\varepsilon}(u) \leq \varepsilon_{1}^{2}(\delta), \\
\left\|H_{r}\right\|_{L_{t}^{\infty} L_{x}^{2}\left(P_{1}\right)}^{2}=r\|H\|_{L_{t}^{\infty} L_{x}^{2}\left(P_{r}(0,1)\right)}^{2} \leq L^{2} r \leq \varepsilon_{1}^{2}(\delta),
\end{gathered}
$$

as $r \leq \frac{\varepsilon_{1}^{2}(\delta)}{L^{2}}$. From this scaling argument, we may further assume that $r=1$ and

$$
\|H\|_{L_{t}^{\infty} L_{x}^{2}\left(P_{1}(0,1)\right)} \leq \varepsilon_{1}(\delta) .
$$

Now we write

$$
\int_{P_{1 / 8}(0,1)} e_{\varepsilon}(u)=\mathrm{I}+\mathrm{II}
$$

with

$$
\mathrm{I}=\int_{\left(1-(1 / 8)^{2}, 1\right) \cap G_{(0,1), 1 / 2}^{\Lambda}} \int_{B_{1 / 8}} e_{\varepsilon}(u), \quad \mathrm{II}=\int_{\left(1-(1 / 8)^{2}, 1\right) \cap B_{(0,1), 1 / 2}^{\Lambda}} \int_{B_{1 / 8}} e_{\varepsilon}(u) .
$$

By (4-3) and Lemma 2.6, we have the estimate

$$
\mathrm{II} \leq\left|B_{(0,1), 1 / 2}^{\Lambda}\right| \sup _{\substack{t \in B_{(0,1), 1 / 2}^{\Lambda} \\ t \in\left[1-(1 / 8)^{2}, 1\right]}} \int_{B_{1 / 8}} e_{\varepsilon}(u) \leq \frac{1}{\Lambda^{2}} \int_{P_{1}(0,1)}\left(e_{\varepsilon}(u)+|H|^{2}\right) .
$$

To estimate I, observe that (5-3) and Lemma 4.2 imply that

$$
|u|(x, t) \geq \frac{1}{2} \quad \text { for all } x \in B_{1 / 4} \text { and } t \in G_{(0,1), 1 / 2}^{\Lambda} .
$$


This, combined with the fact $|u| \leq 1$ in $\Omega \times \mathbb{R}_{+}$, implies

$$
|\nabla u|^{2} \leq 4|u|^{2}|\nabla u|^{2}=4|\nabla u \times u|^{2}+\left.\left.|\nabla| u\right|^{2}\right|^{2} \leq 4\left(|\nabla u \times u|^{2}+|\nabla| u||^{2}\right) .
$$

Therefore $\int_{B_{1 / 8}} e_{\varepsilon}(u) \leq \mathrm{III}+\mathrm{IV}$ for $t \in G_{(0,1), 1 / 2}^{\Lambda}$, with

$$
\mathrm{III}=2 \int_{B_{1 / 8}}|\nabla u \times u|^{2}, \quad \mathrm{IV}=+\int_{B_{1 / 8}}\left(2|\nabla| u||^{2}+\frac{\left(1-|u|^{2}\right)^{2}}{4 \varepsilon^{2}}\right) .
$$

By the definition of $G_{(0,1), 1 / 2}^{\Lambda}$ and Lemma 2.6, we have

$$
\begin{aligned}
\int_{B_{1 / 2}} e_{\varepsilon}(u)+\int_{B_{1 / 2}}\left|\frac{\partial u}{\partial t}\right|^{2} & \leq C \Lambda^{2}\left(\int_{P_{1}(0,1)} e_{\varepsilon}(u)+\int_{P_{1}(0,1)}|H|^{2}\right) \\
& \leq C \Lambda^{2}\left(\int_{P_{1}(0,1)} e_{\varepsilon}(u)+\|H\|_{L_{t}^{\infty} L_{x}^{2}\left(P_{1}(0,1)\right)}^{2}\right) .
\end{aligned}
$$

Hence, for $t \in G_{(0,1), 1 / 2}^{\Lambda}$,

$$
\begin{aligned}
\sup _{x \in B_{1 / 4}}\left\{\int_{B_{1 / 4}(x)} e_{\varepsilon}(u)+\int_{B_{1 / 4}(x)}\left|\frac{\partial u}{\partial t}\right|^{2}\right\} \leq C \Lambda^{2} \int_{P_{1}(0,1)} e_{\varepsilon}(u) \\
+C \Lambda^{2}\|H\|_{L_{t}^{\infty} L_{x}^{2}\left(P_{1}(0,1)\right)}^{2}
\end{aligned}
$$

It follows from (5-9) and Lemma 3.1 that

$$
\begin{aligned}
\sup \left\{s^{-1} \int_{B_{s}(x)}|\nabla u|^{2}: x\right. & \left.\in B_{1 / 4}, 0<s<\frac{1}{4}\right\} \\
& \leq C \Lambda^{2} \int_{P_{1}(0,1)} e_{\varepsilon}(u)+C \Lambda^{2}\|H\|_{L_{t}^{\infty} L_{x}^{2}\left(P_{1}(0,1)\right)}^{2}
\end{aligned}
$$

To estimate III, let $\phi \in C_{0}^{\infty}\left(B_{1 / 4}\right)$ be such that $0 \leq \phi \leq 1, \phi \equiv 1$ in $B_{1 / 8}$, and $|\nabla \phi| \leq 128$. Then we have, by integration by parts,

$$
\begin{aligned}
\int_{B_{1 / 8}}|\nabla u \times u|^{2} & \leq \int_{\mathbb{R}^{3}} \phi^{2}|\nabla u \times u|^{2}=\int_{\mathbb{R}^{3}} \phi^{2}(\nabla u \times u) \cdot(\nabla u \times u) \\
& =\int_{\mathbb{R}^{3}} \phi^{2}(\nabla u \times u) \cdot\left(\nabla\left(u-c_{1 / 4}(t)\right) \times u\right) \\
& =\int_{\mathbb{R}^{3}}\left(\phi^{2}(\nabla u \times u) \times \nabla u\right) \cdot\left(u-c_{1 / 4}(t)\right) \\
& -\int_{\mathbb{R}^{3}} \nabla \cdot\left(\phi^{2}(\nabla u \times u)\right) \cdot\left(\left(u-c_{1 / 4}(t)\right) \times u\right),
\end{aligned}
$$

where

$$
c_{r}(t)=\frac{1}{\left|B_{r}\right|} \int_{B_{r}} u(t) \text { for } r>0 .
$$




\section{Setting}

$$
\lambda=\frac{\int_{\mathbb{R}^{3}} \phi^{2}(\nabla u \times u) \times \nabla u}{\int_{\mathbb{R}^{3}} \phi^{2}},
$$

we can rewrite the expression on the last two lines of (5-11) to obtain

$$
\int_{B_{1 / 8}}|\nabla u \times u|^{2}=\mathrm{III}_{1}+\mathrm{III}_{2}+\mathrm{III}_{3},
$$

where

$$
\begin{gathered}
\mathrm{III}_{1}=\int_{\mathbb{R}^{3}} \phi^{2}((\nabla u \times u) \times \nabla u-\lambda) \cdot\left(u-c_{1 / 4}(t)\right), \quad \mathrm{III}_{2}=\lambda \int_{\mathbb{R}^{3}} \phi^{2}\left(u-c_{1 / 4}(t)\right), \\
\mathrm{III}_{3}=-\int_{\mathbb{R}^{3}} \nabla \cdot\left(\phi^{2}(\nabla u \times u)\right) \cdot\left(\left(u-c_{1 / 4}(t)\right) \times u\right) .
\end{gathered}
$$

It follows from Lemma 2.6 that

$$
|\lambda| \leq C \int_{B_{1 / 4}}|\nabla u|^{2} \leq C\left(\int_{P_{1}(0,1)} e_{\varepsilon}(u)+\|H\|_{L_{t}^{\infty} L_{x}^{2}\left(P_{1}(0,1)\right)}^{2}\right)
$$

so that by Hölder's inequality and Poincaré's inequality,

$$
\begin{aligned}
\left|\mathrm{III}_{2}\right| & \leq|\lambda|\left\|u-c_{1 / 4}(t)\right\|_{L^{2}\left(B_{1 / 4}\right)} \\
& \leq C\left(\int_{P_{1}(0,1)} e_{\varepsilon}(u)+\|H\|_{L_{t}^{\infty} L_{x}^{2}\left(P_{1}(0,1)\right)}^{2}\right)\|\nabla u\|_{L^{2}\left(B_{1 / 4}\right)} \\
& \leq C\left(\int_{P_{1}(0,1)} e_{\varepsilon}(u)+\|H\|_{L_{t}^{\infty} L_{x}^{2}\left(P_{1}(0,1)\right)}^{2}\right)^{3 / 2} .
\end{aligned}
$$

To estimate $\mathrm{III}_{3}$, we first note that (1-19) is equivalent to

$$
\nabla \cdot(\nabla u \times u)=\left(R(u)\left(\frac{\partial u}{\partial t}\right)+u \times(u \times H)\right) \times u .
$$

Hence, by using (5-14), (5-10), and Lemma 2.6,

$$
\begin{aligned}
\int_{\mathbb{R}^{3}}\left|\nabla \cdot\left(\phi^{2} \nabla u \times u\right)\right|^{2} & \leq \int_{\mathbb{R}^{3}}\left(|\nabla \phi|^{2}|\nabla u|^{2}+\phi^{2}|\nabla \cdot(\nabla u \times u)|^{2}\right) \\
& \leq C \int_{B_{1 / 4}}|\nabla u|^{2}+C \int_{B_{1 / 4}}\left(\left|\frac{\partial u}{\partial t}\right|^{2}+|H|^{2}\right) \\
& \leq C \Lambda^{2}\left(\int_{P_{1}(0,1)} e_{\varepsilon}(u)+\|H\|_{L_{t}^{\infty} L_{x}^{2}\left(P_{1}(0,1)\right)}^{2}\right) .
\end{aligned}
$$

Therefore, by Hölder's inequality we have, for any $\delta>0$, 
$(5-15)$

$$
\begin{aligned}
\left|\mathrm{III}_{3}\right| & \leq\left\|\nabla \cdot\left(\phi^{2} \nabla u \times u\right)\right\|_{L^{2}\left(\mathbb{R}^{3}\right)}\left\|u-c_{1 / 4}(t)\right\|_{L^{2}\left(B_{1 / 4}\right)} \\
& \leq \frac{\delta}{4}\left(\int_{P_{1}(0,1)} e_{\varepsilon}(u)+\|H\|_{L_{t}^{\infty} L_{x}^{2}\left(P_{1}(0,1)\right)}^{2}\right)+C \frac{\Lambda^{2}}{\delta}\left\|u-c_{1 / 4}(t)\right\|_{L^{2}\left(B_{1 / 4}\right)}^{2}
\end{aligned}
$$

To estimate $\mathrm{III}_{1}$, we utilize the duality between Hardy and BMO spaces (see also [Hélein 1990; Evans 1991; Wang 2006]). First, by the definition of the BMO norm, the Poincaré inequality, and (5-10), we have

$$
\begin{aligned}
\left(u-c_{1 / 4}(t)\right)_{\mathrm{BMO}\left(B_{1 / 4}\right)}^{2} & \leq \sup \left\{s^{-1} \int_{B_{s}(x)}|\nabla u|^{2}: x \in B_{1 / 4}, 0<s<\frac{1}{4}\right\} \\
& \leq C \Lambda^{2}\left(\int_{P_{1}(0,1)} e_{\varepsilon}(u)+\|H\|_{L_{t}^{\infty} L_{x}^{2}\left(P_{1}(0,1)\right)}^{2}\right) .
\end{aligned}
$$

Therefore by (5-15), (5-16), and [Wang 2006, Propositions 5.6-5.8], we have

$$
\begin{aligned}
\left|\mathrm{III}_{1}\right| & =\left|\int_{\mathbb{R}^{3}} \phi^{2}((\nabla u \times u) \times \nabla u-\lambda) \cdot\left(u-c_{1 / 4}(t)\right)\right| \\
& \leq C\left\|\phi^{2}((\nabla u \times u) \times \nabla u-\lambda)\right\|_{\mathscr{H}^{1}\left(\mathbb{R}^{3}\right)} u-c_{1 / 4}(t)_{\mathrm{BMO}\left(B_{1 / 4}\right)} \\
& \leq C\left\|\phi^{2}(\nabla u \times u) \times \nabla u\right\|_{\mathscr{H}^{1}\left(B_{1 / 4}, B_{1 / 2}\right)}\left(u-c_{1 / 4}(t)\right)_{\mathrm{BMO}\left(B_{1 / 4}\right)} \\
& \leq C\left(u-c_{1 / 4}(t)\right)_{\mathrm{BMO}\left(B_{1 / 4}\right)}\left(\|\nabla u\|_{L^{2}\left(B_{1 / 2}\right)}^{2}+\|\nabla \cdot(\nabla u \times u)\|_{L^{2}\left(B_{1 / 2}\right)}^{2}\right) \\
& \leq C \Lambda^{3}\left(\int_{P_{1}(0,1)} e_{\varepsilon}(u)+\|H\|_{L_{t}^{\infty} L_{x}^{2}\left(P_{1}(0,1)\right)}^{2}\right)^{3 / 2} .
\end{aligned}
$$

Putting the estimates (5-3), (5-13), (5-15) and (5-17) together, we get

$$
\begin{aligned}
\int_{B_{1 / 8}}|\nabla u \times u|^{2} \leq\left(C \Lambda^{3} \varepsilon_{1}(\delta)+\frac{\delta}{4}\right)\left(\int_{P_{1}(0,1)} e_{\varepsilon}(u)+\|H\|_{L_{t}^{\infty} L_{x}^{2}\left(P_{1}(0,1)\right)}^{2}\right) \\
+C \frac{\Lambda^{2}}{\delta} \int_{B_{1 / 4}}\left|u-c_{1 / 4}(t)\right|^{2}
\end{aligned}
$$

Now we estimate IV as follows. It follows from (5-6) that we can write $u=\rho \omega$, with $\rho=|u| \geq \frac{1}{2}$ and $\omega=u /|u|$. Then $\rho$ satisfies

$$
\Delta \rho-\rho|\nabla \omega|^{2}+\frac{\left(1-\rho^{2}\right) \rho}{\varepsilon^{2}}=R(u)\left(\frac{\partial u}{\partial t}\right) \cdot \omega \quad \text { in } B_{1 / 4} .
$$

Multiplying (5-19) by $\phi^{2}(1-\rho)$ for $\phi \in C_{0}^{\infty}\left(B_{1 / 4}\right)$ and integrating over $B_{1 / 4}$, we can write

$$
\int_{B_{1 / 4}} \phi^{2}\left(\left.\nabla \rho\right|^{2}+\frac{(1-\rho)^{2}}{\varepsilon^{2}} \rho(1+\rho)\right)=\mathrm{IV}_{1}+\mathrm{IV}_{2}+\mathrm{IV}_{3}
$$


where

$$
\begin{gathered}
\mathrm{IV}_{1}=\int_{B_{1 / 4}}(1-\rho) \nabla \rho \cdot \nabla \phi^{2}, \quad \operatorname{IV}_{2}=\int_{B_{1 / 4}} \phi^{2}(1-\rho) R(u)\left(\frac{\partial u}{\partial t}\right) \cdot \omega \\
\operatorname{IV}_{3}=\int_{B_{1 / 4}} \phi^{2} \rho(1-\rho)|\nabla \omega|^{2} .
\end{gathered}
$$

Since $|\nabla \rho| \leq|\nabla u|$, Lemma 2.6 gives

$$
\begin{aligned}
\left|\mathrm{IV}_{1}\right| & \leq \int_{B_{1 / 4}}|\nabla u|\left(1-|\rho|^{2}\right) \leq \varepsilon\left(\int_{B_{1 / 4}}|\nabla u|^{2}\right)^{1 / 2}\left(\int_{B_{1 / 4}} \frac{\left(1-|u|^{2}\right)^{2}}{\varepsilon^{2}}\right)^{1 / 2} \\
& \leq C \Lambda^{2} \varepsilon\left(\int_{P_{1}(0,1)} e_{\varepsilon}(u)+\|H\|_{L_{t}^{\infty} L_{x}^{2}\left(P_{1}(0,1)\right)}\right) .
\end{aligned}
$$

For $\mathrm{IV}_{2}$, we have

$$
\begin{aligned}
\left|\mathrm{IV}_{2}\right| & \leq \int_{B_{1 / 4}}\left|\frac{\partial u}{\partial t}\right|\left(1-|\rho|^{2}\right) \leq \varepsilon\left(\int_{B_{1 / 4}}\left|\frac{\partial u}{\partial t}\right|^{2}\right)^{1 / 2}\left(\int_{B_{1 / 4}} \frac{\left(1-|u|^{2}\right)^{2}}{\varepsilon^{2}}\right)^{1 / 2} \\
& \leq C \Lambda^{2} \varepsilon\left(\int_{P_{1}(0,1)} e_{\varepsilon}(u)+\|H\|_{L_{t}^{\infty} L_{x}^{2}\left(P_{1}^{+}(0,1)\right)}^{2}\right)
\end{aligned}
$$

Since $|\omega|=1$ and $\rho \geq \frac{1}{2}$, we have $|\nabla \omega|^{2} \leq 14|\nabla u \times u|^{2}$. Hence

$$
\left|\mathrm{IV}_{3}\right| \leq C \int_{B_{1 / 4}}|\nabla u \times u|^{2} .
$$

Therefore, for $t \in G_{(0,1), 1 / 2}^{\Lambda}$,

(5-24) $\quad|\mathrm{IV}| \leq C \Lambda^{2} \varepsilon\left(\int_{P_{1}(0,1)} e_{\varepsilon}(u)+\|H\|_{L_{t}^{\infty} L_{x}^{2}\left(P_{1}(0,1)\right)}^{2}\right)+C \int_{B_{1 / 4}}|\nabla u \times u|^{2}$.

Putting the estimates for III and IV together, we obtain for any $t \in G_{(0,1), 1 / 2}^{\Lambda}$,

$$
\begin{array}{r}
\int_{B_{1 / 8}} e_{\varepsilon}(u) \leq\left(C \Lambda^{2}\left(\varepsilon+\Lambda \varepsilon_{1}(\delta)\right)+\frac{\delta}{4}\right)\left(\int_{P_{1}(0,1)} e_{\varepsilon}(u)\right. \\
\left.+\|H\|_{L_{t}^{\infty} L_{x}^{2}\left(P_{1}(0,1)\right)}^{2}\right) \\
+C \frac{\Lambda^{2}}{\delta} \int_{B_{1}}\left|u-c_{1}(t)\right|^{2}
\end{array}
$$

Integrating (5-25) over $t \in G_{(0,1), 1 / 2}^{\Lambda}$ and adding (5-5), we obtain

(5-26) $\left(\frac{1}{8}\right)^{-3} \int_{P_{1 / 8}(0,1)} e_{\varepsilon}(u)$

$$
\begin{array}{r}
\leq\left(C \Lambda^{2}\left(\varepsilon+\Lambda \varepsilon_{1}(\delta)\right)+\frac{\delta}{4}+\frac{1}{\Lambda^{2}}\right)\left(\int_{P_{1}(0,1)} e_{\varepsilon}(u)+\|H\|_{L_{t}^{\infty} L_{x}^{2}\left(P_{1}(0,1)\right)}^{2}\right) \\
+\frac{C \Lambda^{2}}{\delta} \int_{P_{1}(0,1)}\left|u-c_{1}(t)\right|^{2} .
\end{array}
$$


Lemma 5.1 will be proved if we choose, for any fixed small $\delta>0$, a sufficiently large $\Lambda=2 / \sqrt{\delta}>0$, a sufficiently small $\varepsilon=\delta /(16 C)$ and $\varepsilon_{1}(\delta)=\delta^{5 / 2} /(32 C)$. Here we have also used in the last step the fact that

$$
\int_{P_{1}(0,1)}\left|u-c_{1}(t)\right|^{2} \leq 2 \int_{P_{1}(0,1)}\left|u-u_{P_{1}(0,1)}\right|^{2} .
$$

Lemma 5.2. There exists a constant $C_{0}>0$ such that for any $L>0, \theta \in\left(0, \frac{1}{4}\right)$ there are $\varepsilon(\theta), \varepsilon_{1}(\theta)>0$ such that if $\left(u^{\varepsilon}, H^{\varepsilon}\right)$ is the weak solution of (1-19) in Lemma 2.2 and we take $0<r<\min \left\{\operatorname{dist}\left(x_{0}, \partial \Omega\right), \sqrt{t_{0}}, \varepsilon_{1}^{2}(\theta) / L^{2}\right\}, \varepsilon<\varepsilon(\theta) r$, and $z_{0}=\left(x_{0}, t_{0}\right) \in \Omega \times \mathbb{R}_{+}$satisfying

$$
\left\|H^{\varepsilon}\right\|_{L_{t}^{\infty} L_{x}^{2}\left(P_{r}\left(z_{0}\right)\right)} \leq L \quad \text { and } \quad \int_{P_{r}\left(z_{0}\right)} e_{\varepsilon}\left(u^{\varepsilon}\right) \leq \varepsilon_{1}^{2}(\theta),
$$

then

$$
\frac{1}{(\theta r)^{5}} \int_{P_{\theta r}\left(z_{0}\right)}\left|u^{\varepsilon}-u_{P_{\theta r}\left(z_{0}\right)}^{\varepsilon}\right|^{2} \leq C_{0} \theta^{2} \max \left\{r^{-3} \int_{P_{r}\left(z_{0}\right)} e_{\varepsilon}\left(u^{\varepsilon}\right), r\left\|H^{\varepsilon}\right\|_{L_{t}^{\infty} L_{x}^{2}\left(P_{r}\left(z_{0}\right)\right)}^{2}\right\}
$$

where $u_{P_{\theta r}\left(z_{0}\right)}^{\varepsilon}$ is the average of $u^{\varepsilon}$ over $P_{\theta r}\left(z_{0}\right)$.

Proof. Write $(u, H)$ for $\left(u^{\varepsilon}, H^{\varepsilon}\right)$. Assume that $z_{0}=(0,1), r=1$, and

$$
\|H\|_{L_{t}^{\infty} L_{x}^{2}\left(P_{1}(0,1)\right)} \leq \varepsilon_{1}(\theta) .
$$

Now we argue by contradiction. Suppose that Lemma 5.2 is false. Then there are $\theta_{0} \in\left(0, \frac{1}{4}\right), \varepsilon_{k} \downarrow 0$, and a sequence of weak solutions $\left(u^{k}, H^{k}\right)$ of (1-19) corresponding to $\varepsilon=\varepsilon_{k}$ such that

$$
\int_{P_{1}(0,1)} e_{\varepsilon_{k}}\left(u^{k}\right)=\delta_{k}^{2} \downarrow 0, \quad\left\|H^{k}\right\|_{L_{t}^{\infty} L^{2}\left(P_{1}(0,1)\right)}^{2} \leq \delta_{k}^{2},
$$

but

$$
\theta_{0}^{-5} \int_{P_{\theta_{0}}(0,1)}\left|u^{k}-u_{P_{\theta_{0}}(0,1)}^{k}\right|^{2} \geq k \theta_{0}^{2} \max \left\{\int_{P_{1}(0,1)} e_{\varepsilon_{k}}\left(u^{k}\right),\left\|H^{k}\right\|_{L_{t}^{\infty} L^{2}\left(P_{1}(0,1)\right)}^{2}\right\} .
$$

Define

$$
v^{k}=\frac{u^{k}-u_{P_{1}(0)}^{k}}{\delta_{k}}
$$

By Lemma 2.6, $\left\{v^{k}\right\}$ is uniformly bounded in $H^{1}\left(P_{1 / 2}(0,1)\right)$ and $\left(v^{k}\right)_{P_{1}(0,1)}=0$. Assume that $v^{k} \rightarrow v$ weakly in $H^{1}\left(P_{1 / 2}(0,1), \mathbb{R}^{3}\right)$, strongly in $L^{2}\left(P_{1 / 2}(0,1), \mathbb{R}^{3}\right)$, and $u^{k} \rightarrow p$ for some $p \in S^{2}$. It is not hard to show that $v \in T_{p} S^{2}$ and hence we have $R(p)(\partial v / \partial t)-\Delta v \in T_{p} S^{2}$. Observe that

$$
\left(R\left(u^{k}\right)\left(\frac{\partial v^{k}}{\partial t}\right)-\Delta v^{k}-\delta_{k}^{-1}\left(u^{k} \times\left(H^{k} \times u^{k}\right)\right)\right) \times u^{k}=0,
$$


and (5-29) implies

$$
\left|\delta_{k}^{-1}\left(u^{k} \times\left(H^{k} \times u^{k}\right)\right) \times u^{k}\right| \leq \frac{\left|H^{k}\right|}{\delta_{k}} \rightarrow 0 \quad \text { in } L^{2}\left(P_{1}(0,1)\right) \text { as } k \rightarrow \infty .
$$

By sending $k$ to $\infty$, we conclude that $v$ solves

$$
\left(R(p) \frac{\partial v}{\partial t}-\Delta v\right) \times p=0
$$

Therefore

$$
R(p) \frac{\partial v}{\partial t}-\Delta v=0 \text { in } P_{1 / 2}(0,1) .
$$

Standard parabolic theory [Lieberman 1996] implies

$$
\theta_{0}^{-5} \int_{P_{\theta_{0}}(0,1)}|v|^{2} \leq C \theta_{0}^{2} \int_{P_{1}}|\nabla v|^{2}
$$

which contradicts (5-29).

Combining Lemma 5.1 and Lemma 5.2, we can prove:

Lemma 5.3. For any $\gamma \in(0,1)$, there are $\theta \in\left(0, \frac{1}{4}\right), C_{1}>0, k_{0}>0, \varepsilon_{2}>0$ such that if $\left(u^{\varepsilon}, H^{\varepsilon}\right)$ is the weak solution of (1-19) in Lemma 2.2 and we take $z_{0}=$ $\left(x_{0}, t_{0}\right) \in \Omega \times \mathbb{R}_{+}, L>0,0<r<\min \left\{\operatorname{dist}\left(x_{0}, \partial \Omega\right), \sqrt{t_{0}}, \varepsilon_{2}^{2} / L^{2}\right\}$, and $0<\varepsilon \leq k_{0} r$ satisfying

$$
\left\|H^{\varepsilon}\right\|_{L_{t}^{\infty} L_{x}^{2}\left(P_{r}\left(z_{0}\right)\right)} \leq L \quad \text { and } \quad r^{-3} \int_{P_{r}\left(z_{0}\right)} e_{\varepsilon}\left(u^{\varepsilon}\right) \leq \varepsilon_{2}^{2},
$$

then

$$
(\theta r)^{-3} \int_{P_{\theta r}\left(z_{0}\right)} e_{\varepsilon}\left(u^{\varepsilon}\right) \leq C_{1}\left(\theta^{2 \gamma} r^{-3} \int_{P_{r}\left(z_{0}\right)} e_{\varepsilon}\left(u^{\varepsilon}\right)+\theta r\left\|H^{\varepsilon}\right\|_{L_{t}^{\infty} L_{x}^{2}\left(P_{r}\left(z_{0}\right)\right)}^{2}\right) .
$$

Proof. Again we write $(u, H)$ for $\left(u^{\varepsilon}, H^{\varepsilon}\right)$. As in the proof of Lemmas 5.1 and 5.2, we may assume that $z_{0}=(0,1), r=1$, and

$$
\|H\|_{L_{t}^{\infty} L_{x}^{2}\left(P_{1}(0,1)\right)} \leq \varepsilon_{2} .
$$

Set $\delta=8^{-3}$ and

$$
\theta=\theta(\gamma) \leq\left(\frac{\delta^{2}}{2 C_{0} C(\delta)}\right)^{1 /(2-2 \gamma)} .
$$

Here $C_{0}>0$ and $C(\delta)>0$ are given by Lemma 5.2 and Lemma 5.1 respectively. Let $k \geq 1$ be such that $8^{k} \theta=1$. For $0<\rho<1$, set

$$
E(u, \rho)=\rho^{-3} \int_{P_{\rho}(0,1)} e_{\varepsilon}(u), \quad F(H, \rho)=\rho\|H\|_{L_{t}^{\infty} L_{x}^{2}\left(P_{\rho}(0,1)\right)}^{2} .
$$


For $0 \leq i \leq k-1$, if $E\left(u, 8^{i+1} \theta\right) \leq \varepsilon_{1}^{2}(\delta)$ and $E(u, 1) \leq \varepsilon_{1}^{2}\left(8^{i+1} \theta\right)$, then Lemmas 5.1 and 5.2 imply

(5-34) $\quad E\left(u, 8^{i} \theta\right)$

$$
\leq \delta \max \left\{E\left(u, 8^{i+1} \theta\right), F\left(H, 8^{i+1} \theta\right)\right\}+\frac{C_{0} C(\delta)}{\delta} \max \{E(u, 1), F(H, 1)\} .
$$

Now we choose

$$
\varepsilon_{2} \equiv \frac{\delta}{2 C_{0} C(\delta)} \min \left\{\varepsilon_{1}(8 \theta), \ldots, \varepsilon_{1}\left(8^{k} \theta\right), \varepsilon_{1}(\delta)\right\} .
$$

Since $F(H, \rho) \leq \rho F(H, 1) \leq F(H, 1) \leq \varepsilon_{2}^{2},(5-34)$ implies that

$$
E\left(u, 8^{i} \theta\right) \leq \min \left\{\varepsilon_{1}^{2}(8 \theta), \ldots, \varepsilon_{1}^{2}\left(8^{k} \theta\right), \varepsilon_{1}^{2}(\delta)\right\} \quad \text { for all } 0 \leq i \leq k .
$$

Hence by iteration, (5-34) implies

(5-35) $E(u, \theta)$

$$
\begin{aligned}
& \leq \delta^{k} E(u, 1)+F(H, 1) \sum_{i=1}^{k}(8 \delta \theta)^{i}+\frac{C_{0} C(\delta)}{1-64 \delta}\left(\frac{\theta}{\delta}\right)^{2} \max \{E(u, 1), F(H, 1)\} \\
& \leq \delta^{k} E(u, 1)+\frac{8 \delta \theta}{1-8 \delta \theta} F(H, 1)+\frac{C_{0} C(\delta)}{1-64 \delta}\left(\frac{\theta}{\delta}\right)^{2} \max \{E(u, 1), F(H, 1)\} .
\end{aligned}
$$

According to the definition, $\delta^{k}=\theta^{3}$ and $2 C_{0} C(\delta) / \delta^{2} \leq \theta^{2-2 \gamma}$. So (5-35) gives $E(u, \theta) \leq \max \left\{C_{1} \theta^{2 \gamma} E(u, 1), C_{1} \theta F(H, 1)\right\}$, which clearly implies (5-32).

The following proposition plays a crucial role in the proof of Theorem 1.2:

Proposition 5.4. For any $u_{0} \in H^{1}\left(\Omega, S^{2}\right), H_{0} \in L^{2}\left(\mathbb{R}^{3}, \mathbb{R}^{3}\right), E_{0} \in L^{2}\left(\mathbb{R}^{3}, \mathbb{R}^{3}\right)$, $\varepsilon>0$ and $0<T<+\infty$, let

$$
\left(u^{\varepsilon}, H^{\varepsilon}, E^{\varepsilon}\right) \in H^{1}\left(\Omega \times[0, T], \mathbb{R}^{3}\right) \times L^{2}\left(\mathbb{R}^{3} \times[0, T], \mathbb{R}^{3}\right) \times L^{2}\left(\mathbb{R}^{3} \times[0, T], \mathbb{R}^{3}\right)
$$

be the weak solution of (1-19)+(1-2)-(1-3) under conditions (1-8)-(1-11) obtained in Lemma 2.2. Then there exist universal constants $k_{0}>0, \varepsilon_{3}>0, C_{2}>0$, such that for any $z_{0}=\left(x_{0}, t_{0}\right) \in \Omega \times \mathbb{R}_{+}, 0<r<\min \left\{\operatorname{dist}\left(x_{0}, \partial \Omega\right), \sqrt{t_{0}}, \varepsilon_{3}^{2} / C_{2}\right\}$, if

$$
\mathscr{E}\left(u^{\varepsilon}, z_{0}, r\right) \equiv r^{-3} \int_{P_{r}\left(z_{0}\right)} e_{\varepsilon}\left(u^{\varepsilon}\right) \leq \varepsilon_{3}^{2},
$$

then for any $z \in P_{r / 2}\left(z_{0}\right), \varepsilon / k_{0} \leq \rho \leq \frac{1}{4} r$,

$$
\rho^{-3} \int_{P_{\rho}(z)}\left(e_{\varepsilon}\left(u^{\varepsilon}\right)+\rho^{2}\left|\frac{\partial u^{\varepsilon}}{\partial t}\right|^{2}\right) \leq C_{2} \frac{\rho}{r} \max \left\{\mathscr{E}\left(u^{\varepsilon}, z_{0}, r\right), r\left\|H^{\varepsilon}\right\|_{L_{t}^{\infty} L_{x}^{2}\left(P_{r}\left(z_{0}\right)\right)}^{2}\right\}
$$


Proof. By (2-8) of Lemma 2.2, we have $H^{\varepsilon} \in L^{\infty}\left([0, T], L^{2}\left(\mathbb{R}^{3}\right)\right)$ and

$$
\left\|H^{\varepsilon}\right\|_{L_{t}^{\infty} L_{x}^{2}\left(\mathbb{R}^{3} \times[0, T]\right)} \leq e^{C T}\left(\int_{\Omega}\left|\nabla u_{0}\right|^{2}+\int_{\mathbb{R}^{3}}\left(\varepsilon_{0}\left|E_{0}\right|^{2}+\left|H_{0}\right|^{2}\right)\right) \equiv C_{2} .
$$

This implies that for any $0<\rho \leq r$ and $z \in P_{r / 2}\left(z_{0}\right)$

$$
\rho\left\|H^{\varepsilon}\right\|_{L_{t}^{\infty} L_{x}^{2}\left(P_{\rho}(z)\right)} \leq r\left\|H^{\varepsilon}\right\|_{L_{t}^{\infty} L_{x}^{2}\left(\mathbb{R}^{3} \times[0, T]\right)} \leq r C_{2} \leq \varepsilon_{3}^{2} .
$$

Choose $\varepsilon_{3} \leq \varepsilon_{2}$, where $\varepsilon_{2}$ is given by Lemma 5.3. Then the condition (5-31) of Lemma 5.3 is satisfied for $P_{r / 2}(z)$ with $z \in P_{r / 2}\left(z_{0}\right)$. Hence we can repeatedly apply Lemma 5.3 with $\gamma=\frac{1}{2}$ to obtain that for $0<\rho<\frac{r}{4}, \varepsilon \leq k_{0} \rho$,

$$
\mathscr{E}\left(u^{\varepsilon}, z, \rho\right) \leq C_{1} \frac{\rho}{r} \max \left\{\mathscr{E}\left(u^{\varepsilon}, z_{0}, r\right), r\left\|H^{\varepsilon}\right\|_{L_{t}^{\infty} L_{x}^{2}\left(P_{r}\left(z_{0}\right)\right)}^{2}\right\} .
$$

This, combined with Lemma 2.6, implies (5-37).

Proof of Theorem 1.2. For $\varepsilon>0$, let $\left(u^{\varepsilon}, H^{\varepsilon}, E^{\varepsilon}\right)$ be the weak solution of the system (1-19)+(1-2)-(1-3) with conditions (1-8)-(1-11) obtained in Lemma 2.2. It follows from (2-8) that we may assume that $u^{\varepsilon} \rightarrow u$ weakly in $H_{\text {loc }}^{1}\left(\Omega \times \mathbb{R}_{+}, \mathbb{R}^{3}\right)$, $\left(H^{\varepsilon}, E^{\varepsilon}\right) \rightarrow(H, E)$ weakly in $L_{\text {loc }}^{2}\left(\mathbb{R}^{3} \times \mathbb{R}_{+}, \mathbb{R}^{3}\right)$. By the argument in [Carbou and Fabrie 1998], we know that $(u, H, E)$ is a weak solution of the Landau-LifshitzMaxwell system (1-13)+(1-2)-(1-3) under the initial-boundary conditions (1-8)(1-11).

Now we want to show partial regularity of $u$ as follows. Let $\varepsilon_{3}$ be given by Proposition 5.4, and define the concentrate set of $u^{\varepsilon}$ by

$$
\Sigma=\bigcap_{r>0}\left\{z \in \Omega \times \mathbb{R}^{+}: \liminf _{\varepsilon \rightarrow 0} r^{-3} \int_{P_{r}(z)} e_{\varepsilon}\left(u^{\varepsilon}\right) \geq \varepsilon_{3}^{2}\right\} .
$$

Then a standard covering argument (see [Chen and Struwe 1989]) shows that $\mathscr{P}^{3}(\Sigma \cap K)<\infty$ for any compact subset of $\Omega \times \mathbb{R}^{+}$. Since $u$ is a weak limit in $H_{\mathrm{loc}}^{1}\left(\Omega \times \mathbb{R}_{+}, \mathbb{R}^{3}\right)$ of $u_{\varepsilon}$ as $\varepsilon \downarrow 0$, we conclude that for any $z_{0} \in \Omega \times \mathbb{R}_{+} \backslash \Sigma$, the lower semicontinuity, (5-40), and Proposition 5.4 imply that there exists $r_{0}>0$ such that for any $z \in P_{r / 2}\left(z_{0}\right)$ and $0<\rho \leq \frac{1}{4} r$,

$$
\rho^{-3} \int_{P_{\rho}(z)}\left(|\nabla u|^{2}+\rho^{2}\left|\frac{\partial u}{\partial t}\right|^{2}\right) \leq C_{3} \frac{\rho}{r}
$$

for some universal constant $C_{3}>0$. This implies that $u \in C^{1 / 2}\left(\Omega \times \mathbb{R}_{+} \backslash \Sigma, S^{2}\right)$, by the parabolic version of Morrey's Lemma [Chen et al. 1995]. This completes the proof of Theorem 1.2. 


\section{6. $C^{\alpha}$-regularity of $\nabla u$ and proofs of Theorems 1.3 and 1.4}

This section is devoted to the discussion of partial $C^{\alpha}$-regularity of $\nabla u$, when $(u, H, E)$ is a weak solution of (1-13)+(1-2)-(1-3) obtained as in Theorem 1.2 in two special cases: (i) either $\varepsilon_{0}=0$ in (1-2) or (ii) $\beta=0$ in (1-3). For case (i), we assume that the initial data $\left(u_{0}, H_{0}\right) \in H^{1}\left(\Omega, S^{2}\right) \times H^{1}\left(\mathbb{R}^{3}, \mathbb{R}^{3}\right)$ and $H_{0}$ satisfies $\nabla \cdot\left(H_{0}+\beta u_{0}\right)=0$. For case (ii), we assume that the initial data $\left(u_{0}, H_{0}, E_{0}\right) \in$ $H^{1}\left(\Omega, S^{2}\right) \times H^{1}\left(\mathbb{R}^{3}, \mathbb{R}^{3}\right) \times H^{1}\left(\mathbb{R}^{3}, \mathbb{R}^{3}\right)$ and $H_{0}, E_{0}$ satisfy $\nabla \cdot H_{0}=\nabla \cdot E_{0}=0$.

There are two steps to proving $C^{\alpha}$-regularity of $\nabla u$ in $\Omega \times \mathbb{R}_{+} \backslash \Sigma$, where $\Sigma$ is the concentration set (5-40). The first step is to use $H \in L_{t}^{\infty} L_{x}^{6}\left(\mathbb{R}^{3} \times[0, T]\right)$ for any $0<T<+\infty$ to show that $u \in C^{\gamma}\left(\Omega \times \mathbb{R}_{+} \backslash \Sigma, S^{2}\right)$ for any $\gamma \in(0,1)$. The second step is to employ a parabolic hole-filling technique similar to [Giaquinta and Hildebrandt 1982; Giaquinta and Struwe 1982] to show that for $z \in \Omega \times \mathbb{R}_{+} \backslash \Sigma$,

$$
\rho^{-5} \int_{P_{\rho}(z)}\left|\nabla u-(\nabla u)_{P_{\rho}(z)}\right|^{2} \leq C \rho^{2 \alpha} \quad \text { for some } \alpha \in(0,1) .
$$

This can be summarized as follows.

Lemma 6.1. For any $u_{0} \in H^{1}\left(\Omega, S^{2}\right), H_{0} \in H^{1}\left(\mathbb{R}^{3}, \mathbb{R}^{3}\right)$, and $0<T<+\infty$, let $(u, H) \in H^{1}\left(\Omega \times[0, T], S^{2}\right) \times L_{t}^{\infty} L_{x}^{2}\left(\mathbb{R}^{3} \times[0, T], \mathbb{R}^{3}\right)$ be a weak solution to $(1-13)$ coupled with (1-17) under the initial-boundary conditions (1-8)-(1-10) obtained as the weak limit of $\left(u^{\varepsilon}, H^{\varepsilon}\right)$ given by Lemma 2.3. Let $\Sigma \subset \Omega \times \mathbb{R}_{+}$be defined by (5-40). For any $z_{0} \in \Omega \times \mathbb{R}_{+} \backslash \Sigma$, there exists $r_{0}>0$ such that $\nabla u \in C^{\alpha}\left(P_{r_{0}}\left(z_{0}\right)\right)$ for some $\alpha \in(0,1)$.

Proof. By (2-17) of Lemma 2.3,

$$
\begin{aligned}
\sup _{\varepsilon>0}\left(\left\|H^{\varepsilon}\right\|_{L_{t}^{\infty} L_{x}^{2}\left(\mathbb{R}^{3} \times[0, T]\right)}\right. & \left.+\left\|\nabla H^{\varepsilon}\right\|_{L_{t}^{\infty} L_{x}^{2}\left(\mathbb{R}^{3} \times[0, T]\right)}\right) \\
& \leq e^{C T}\left(\left\|\nabla u_{0}\right\|_{L^{2}(\Omega)}^{2}+\left\|H_{0}\right\|_{L^{2}\left(\mathbb{R}^{3}\right)}^{2}+\left\|\nabla H_{0}\right\|_{L^{2}\left(\mathbb{R}^{3}\right)}^{2}\right) .
\end{aligned}
$$

By the Sobolev embedding theorem, (6-1) implies $H^{\varepsilon} \in L_{t}^{\infty} L_{x}^{6}\left(\mathbb{R}^{3} \times[0, T]\right)$ and

$$
\sup _{\varepsilon>0}\left\|H^{\varepsilon}\right\|_{L_{t}^{\infty} L_{x}^{6}\left(\mathbb{R}^{3} \times[0, T]\right)} \leq C_{3} \equiv C\left(T,\left\|u_{0}\right\|_{H^{1}(\Omega)},\left\|H_{0}\right\|_{H^{1}\left(\mathbb{R}^{3}\right)}\right) .
$$

Since $z_{0} \in \Omega \times \mathbb{R}_{+} \backslash \Sigma$, by (5-40), there exists $0<r_{0} \leq \varepsilon_{3}^{2} / C_{3}^{2}$ such that $\mathscr{E}\left(u^{\varepsilon}, z_{0}, r_{0}\right) \equiv r_{0}^{-3} \int_{P_{r_{0}}\left(z_{0}\right)} e_{\varepsilon}\left(u^{\varepsilon}\right) \leq \varepsilon_{3}^{2}, \quad \mathscr{F}\left(H^{\varepsilon}, z_{0}, r_{0}\right) \equiv r_{0}\left\|H^{\varepsilon}\right\|_{L_{t}^{\infty} L_{x}^{2}\left(P_{r_{0}}\left(z_{0}\right)\right)}^{2} \leq \varepsilon_{3}^{2}$.

Hence we can apply Lemma 5.3 to conclude that for any $\theta \in\left(0, \frac{1}{2}\right), \gamma \in(0,1)$, $z \in P_{r_{0} / 2}\left(z_{0}\right)$ and $0<r<r_{0} / 2$, there is $C_{4}>0$ such that

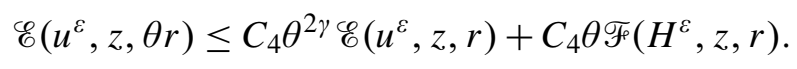


By Hölder's inequality we have

$$
\mathscr{F}\left(H^{\varepsilon}, z, r\right) \leq r^{3}\left\|H^{\varepsilon}\right\|_{L_{t}^{\infty} L_{x}^{2}\left(P_{r}(z)\right)} \leq C_{3} r^{3} \quad \text { for all } 0<r \leq r_{0} .
$$

Therefore (6-3) yields, for $z \in P_{r_{0} / 2}\left(z_{0}\right)$ and $0<r<r_{0} / 2$,

$$
\mathscr{E}\left(u^{\varepsilon}, z, \theta r\right) \leq C_{5}\left(\theta^{2 \gamma \mathscr{E}}\left(u^{\varepsilon}, z, r\right)+\theta r^{3}\right) .
$$

Iterating (6-4) $k$ times, we obtain

$$
\begin{aligned}
\mathscr{E}\left(u^{\varepsilon}, z, \theta^{k} r\right) & \leq\left(C_{5} \theta^{2 \gamma}\right)^{k \mathscr{E}}\left(u^{\varepsilon}, z, r\right)+\left(\sum_{i=0}^{k-1}\left(C_{5} \theta^{2 \gamma}\right)^{k-1-i}\left(\theta^{3}\right)^{i}\right) r^{3} \\
& \leq\left(C_{5} \theta^{2 \gamma}\right)^{k}\left(\mathscr{E}\left(u^{\varepsilon}, z, r\right)+\frac{r^{3}}{C_{5} \theta^{2 \gamma}-\theta^{3}}\right) .
\end{aligned}
$$

In particular,

$$
\mathscr{E}\left(u^{\varepsilon}, z, s\right) \leq\left(\frac{s}{r_{0}}\right)^{2 \gamma}\left(\mathscr{E}\left(u^{\varepsilon}, z, \frac{r_{0}}{2}\right)+C_{6} r_{0}^{3}\right) \quad \text { for all } z \in P_{r_{0} / 2}\left(z_{0}\right), 0<s \leq \frac{r_{0}}{2} .
$$

In view of Lemma 2.6 and taking $\varepsilon \downarrow 0$, this implies that for $z \in P_{r_{0} / 2}\left(z_{0}\right)$ and $0<s \leq r_{0} / 2$,

$$
s^{-3} \int_{P_{s}(z)}\left(|\nabla u|^{2}+s^{2}\left|\frac{\partial u}{\partial t}\right|^{2}\right) \leq\left(\frac{s}{r_{0}}\right)^{2 \gamma}\left(\varepsilon_{3}^{2}+C_{6} r_{0}^{3}\right) .
$$

Hence the parabolic version of Morrey's Lemma implies that $u \in C^{\gamma}\left(P_{r_{0} / 2}\left(z_{0}\right), S^{2}\right)$ for any $0<\gamma<1$, and

$$
\operatorname{osc}_{P_{r}\left(z_{0}\right)} u \leq C\left(\frac{r}{r_{0}}\right)^{\gamma}\left(\varepsilon_{3}^{2}+C_{6} r_{0}^{3}\right), \quad 0<r \leq \frac{r_{0}}{2} .
$$

Next we will use a parabolic hole-filling argument to show $\nabla u \in C^{\alpha}\left(P_{r_{0} / 2}\left(z_{0}\right)\right)$ for some $\alpha \in(0,1)$. The linear map $R(u) \xi=\alpha_{1} \xi+\alpha_{2} u \times \xi: \mathbb{R}^{3} \rightarrow \mathbb{R}^{3}$ can be represented by

$$
R(u)=\left(\begin{array}{ccc}
\alpha_{1} & -\alpha_{2} u_{3} & \alpha_{2} u_{2} \\
\alpha_{2} u_{3} & \alpha_{1} & -\alpha_{2} u_{1} \\
-\alpha_{2} u_{2} & \alpha_{2} u_{1} & \alpha_{1}
\end{array}\right) .
$$

It is easy to check that $R(u)$ has an inverse $M(u)$, and that this inverse a uniformly elliptic matrix. Now we can rewrite the equation of $u$ as

(6-8) $\frac{\partial u}{\partial t}-\nabla \cdot(M(u) \nabla u)=M(u)\left(|\nabla u|^{2} u+(H-\langle H, u\rangle u)\right)-\nabla(M(u)) \cdot \nabla u$.

For any $z_{1} \in P_{r_{0} / 2}\left(z_{0}\right)$ and $0<r<r_{0} / 2$, consider an auxiliary equation for $v$ : $P_{r}\left(z_{1}\right) \rightarrow \mathbb{R}^{3}:$

$$
\frac{\partial v}{\partial t}-\nabla \cdot\left(M\left(u\left(z_{1}\right)\right) \nabla v\right)=0 \text { in } P_{r}\left(z_{1}\right), \quad v=u \text { on } \partial_{p} P_{r}\left(z_{1}\right),
$$


where $\partial_{p} P_{r}\left(z_{1}\right)$ denotes the parabolic boundary of $P_{r}\left(z_{1}\right)$. It follows from the maximum principle, (6-7), and (6-6) that

$$
\operatorname{osc}_{P_{r}\left(z_{1}\right)} v \leq C_{7} r^{\gamma}, \quad \int_{P_{r}\left(z_{1}\right)}|\nabla v|^{2} \leq \int_{P_{r}\left(z_{1}\right)}|\nabla u|^{2} \leq C_{7} r^{3+2 \gamma} .
$$

Multiplying (6-8) and (6-9) by $w \equiv u-v$ and integrating over $P_{r}\left(z_{1}\right)$, we obtain

$$
\int_{P_{r}\left(z_{1}\right)}\left\langle M\left(u\left(z_{1}\right)\right) \nabla w, \nabla w\right\rangle \leq \mathrm{I}+\mathrm{II},
$$

with I $=C_{8} \int_{P_{r}\left(z_{1}\right)}\left(|\nabla u|^{2}+|H|\right)|w|$ and II $=C_{8} \int_{P_{r}\left(z_{1}\right)}\left|M(u)-M\left(u\left(z_{1}\right)\right)\right||\nabla u||\nabla w|$. By the ellipticity of $M\left(u\left(z_{1}\right)\right)$, we have

$$
\int_{P_{r}\left(z_{1}\right)}\left\langle M\left(u\left(z_{1}\right)\right) \nabla w, \nabla w\right\rangle \geq \alpha_{1} \int_{P_{r}\left(z_{1}\right)}|\nabla w|^{2} .
$$

By Hölder's inequality, (6-7) and (6-10), we have $I \leq C_{9}\left(\frac{r}{r_{0}}\right)^{3+3 \gamma}\left(\varepsilon_{0}^{2}+r_{0}^{3}\right)$, and

$$
\begin{aligned}
\mathrm{II} & \leq \frac{\alpha_{1}}{2} \int_{P_{r}\left(z_{1}\right)}|\nabla u|^{2}+C_{10}\left(\operatorname{osc}_{P_{r}\left(z_{1}\right)} u\right)^{2} \int_{P_{1}\left(z_{1}\right)}|\nabla w|^{2} \\
& \leq \frac{\alpha_{1}}{2} \int_{P_{r}\left(z_{1}\right)}|\nabla u|^{2}+C_{10}\left(\frac{r}{r_{0}}\right)^{3+4 \gamma} .
\end{aligned}
$$

Putting these estimates into (6-11), we obtain

$$
\int_{P_{r}\left(z_{1}\right)}|\nabla w|^{2} \leq C_{11} r^{3+3 \gamma}
$$

Since $v$ solves (6-9), standard parabolic theory implies that for any $0<\rho<r$,

$$
\int_{P_{\rho}\left(z_{1}\right)}\left|\nabla v-(\nabla v)_{P_{\rho}\left(z_{1}\right)}\right|^{2} \leq C_{12}\left(\frac{\rho}{r}\right)^{7} \int_{P_{r}\left(z_{1}\right)}\left|\nabla v-(\nabla v)_{P_{r}\left(z_{1}\right)}\right|^{2} .
$$

Combining (6-13) with (6-14), we obtain that

$$
\begin{aligned}
\int_{P_{\rho}\left(z_{1}\right)}\left|\nabla u-(\nabla u)_{P_{\rho}\left(z_{1}\right)}\right|^{2} & \leq \int_{P_{\rho}\left(z_{1}\right)}\left|\nabla v-(\nabla v)_{P_{\rho}\left(z_{1}\right)}\right|^{2}+\int_{P_{r}\left(z_{1}\right)}|\nabla w|^{2} \\
& \leq C_{12}\left(\frac{\rho}{r}\right)^{7} \int_{P_{r}\left(z_{1}\right)}|\nabla u|^{2}+C_{12} r^{3+3 \gamma}
\end{aligned}
$$

We now choose some $\gamma \in\left(\frac{2}{3}, 1\right)$, whence $3+3 \gamma>5$. Applying the algebraic Lemma 2.1 in [Giaquinta 1983, Chapter III], we conclude that

$$
\rho^{-5} \int_{P_{\rho}\left(z_{1}\right)}\left|\nabla u-(\nabla u)_{P_{\rho}\left(z_{1}\right)}\right|^{2} \leq C_{13} \rho^{3 \gamma-2}\left(1+r^{-(3+3 \gamma)} \int_{P_{r}\left(z_{1}\right)}|\nabla u|^{2}\right)
$$

for any $z_{1} \in P_{r_{0} / 2}\left(z_{0}\right)$ and $0<\rho \leq r \leq r_{0} / 2$. 
A well known characterization of Hölder continuous functions due to Campanato [1965] yields that $\nabla u \in C^{(3 \gamma-2) / 2}\left(P_{r_{0} / 2}\left(z_{0}\right)\right)$. This completes the proof of Lemma 6.1.

Completion of proof of Theorem 1.3. It follows immediately from Lemma 6.1 that $\nabla u \in C^{\alpha}\left(\Omega \times \mathbb{R}_{+} \backslash \Sigma\right)$ for some $\alpha \in(0,1)$. It remains to show that $\nabla^{2} u, \partial u / \partial t \in$ $L_{\text {loc }}^{6}\left(\Omega \times \mathbb{R}_{+} \backslash \Sigma\right)$. To see this, observe that

$$
\begin{aligned}
\left|\frac{\partial u}{\partial t}-\nabla \cdot(M(u) \nabla u)\right| & =\left.|M(u)| \nabla u\right|^{2} u-\nabla(M(u)) \cdot \nabla u+M(u)(H-\langle H, u\rangle u) \mid \\
& \leq C_{14}\left(|\nabla u|^{2}+|H|\right) \in L^{6}\left(P_{R}\right),
\end{aligned}
$$

for any $P_{R} \Subset \Omega \times \mathbb{R}_{+} \backslash \Sigma$. Since $M(u)$ is Hölder continuous and uniformly elliptic, from the $W_{p}^{2,1}$-estimate for the linear parabolic equation [Lieberman 1996], we can conclude that $\nabla^{2} u, \partial u / \partial t \in L^{6}\left(P_{R / 2}\right)$. This implies the second conclusion of Theorem 1.3.

Proof of Theorem 1.4. By applying Lemma 2.4, we can conclude $H^{\varepsilon}$ is bounded in $L_{t}^{\infty} L_{x}^{6}\left(\mathbb{R}^{3} \times[0, T]\right)$ for any $0<T<+\infty$, uniformly in $\varepsilon$. Hence applying the same argument of Lemma 6.1 shows $\nabla u \in C^{\alpha}\left(\Omega \times \mathbb{R}_{+} \backslash \Sigma\right)$ for some $\alpha \in(0,1)$, and $\nabla^{2} u, \partial u / \partial t \in L_{\text {loc }}^{6}\left(\Omega \times \mathbb{R}_{+} \backslash \Sigma\right)$. We leave the details to interested readers.

\section{Acknowledgements}

The first two authors would like to thank Department of Mathematics at the University of Kentucky for its hospitality where most parts of this joint work were conducted. We thank the referee for several useful comments.

\section{References}

[Alouges and Soyeur 1992] F. Alouges and A. Soyeur, "On global weak solutions for LandauLifshitz equations: existence and nonuniqueness", Nonlinear Anal. 18:11 (1992), 1071-1084. MR 93i:35148 Zbl 0788.35065

[Boling and Fengqiu 1997] G. Boling and S. Fengqiu, "Global weak solution for the LandauLifshitz-Maxwell equation in three space dimensions", J. Math. Anal. Appl. 211:1 (1997), 326-346. MR 98e:35159 Zbl 0877.35122

[Campanato 1965] S. Campanato, "Equazioni ellittiche del II deg ordine espazi $\mathfrak{L}^{(2, \lambda) ", ~ A n n . ~ M a t . ~}$ Pura Appl. (4) 69 (1965), 321-381. MR 33 \#395 Zbl 0145.36603

[Carbou and Fabrie 1998] G. Carbou and P. Fabrie, "Time average in micromagnetism”, J. Differential Equations 147:2 (1998), 383-409. MR 2000i:82089 Zbl 0931.35170

[Chen and Lin 1993] Y. M. Chen and F.-H. Lin, "Evolution of harmonic maps with Dirichlet boundary conditions", Comm. Anal. Geom. 1:3-4 (1993), 327-346. MR 95a:58028 Zbl 0845.35049

[Chen and Struwe 1989] Y. M. Chen and M. Struwe, "Existence and partial regularity results for the heat flow for harmonic maps", Math. Z. 201:1 (1989), 83-103. MR 90i:58031 Zbl 0652.58024 
[Chen and Wang 1996] Y. Chen and C. Wang, "Partial regularity for weak heat flows into Riemannian homogeneous spaces", Comm. Partial Differential Equations 21:5-6 (1996), 735-761. MR 98b:58043 Zbl 0861.58034

[Chen et al. 1995] Y. M. Chen, J. Li, and F.-H. Lin, "Partial regularity for weak heat flows into spheres”, Comm. Pure Appl. Math. 48:4 (1995), 429-448. MR 96e:58039 Zbl 0827.35024

[Chen et al. 1998] Y. Chen, S. Ding, and B. Guo, "Partial regularity for two-dimensional LandauLifshitz equations", Acta Math. Sinica (N.S.) 14:3 (1998), 423-432. MR 2000g:35192 Zbl 0906. 35025

[Ding and Guo 2004] S. Ding and B. Guo, "Hausdorff measure of the singular set of Landau-Lifshitz equations with a nonlocal term", Comm. Math. Phys. 250:1 (2004), 95-117. MR 2005g:58034 Zbl 1068.58009

[Ding and Guo 2008] S. Ding and B. Guo, "Existence of partially regular weak solutions to LandauLifshitz-Maxwell equations", J. Differential Equations 244:10 (2008), 2448-2472. MR 2009h: 35210 Zbl 1143.35376

[Ding and Wang 2007] S. Ding and C. Wang, "Finite time singularity of the Landau-Lifshitz-Gilbert equation”, Int. Math. Res. Not. 2007:4 (2007), Art. ID rnm012. MR 2008h:35156 Zbl 1130.35304

[Ding et al. 2007] S. Ding, B. Guo, J. Lin, and M. Zeng, "Global existence of weak solutions for Landau-Lifshitz-Maxwell equations”, Discrete Contin. Dyn. Syst. 17:4 (2007), 867-890. MR 2008a:35274 Zbl 1157.35109

[Evans 1991] L. C. Evans, "Partial regularity for stationary harmonic maps into spheres", Arch. Rational Mech. Anal. 116:2 (1991), 101-113. MR 93m:58026 Zbl 0754.58007

[Feldman 1994] M. Feldman, "Partial regularity for harmonic maps of evolution into spheres", Comm. Partial Differential Equations 19:5-6 (1994), 761-790. MR 95i:58057 Zbl 0807.35021

[Giaquinta 1983] M. Giaquinta, Multiple integrals in the calculus of variations and nonlinear elliptic systems, Annals of Math. Studies 105, Princeton University Press, Princeton, NJ, 1983. MR 86b:49003 Zbl 0516.49003

[Giaquinta and Hildebrandt 1982] M. Giaquinta and S. Hildebrandt, "A priori estimates for harmonic mappings", J. Reine Angew. Math. 336 (1982), 124-164. MR 84b:58035 Zbl 0508.58015

[Giaquinta and Struwe 1982] M. Giaquinta and M. Struwe, "On the partial regularity of weak solutions of nonlinear parabolic systems”, Math. Z. 179:4 (1982), 437-451. MR 83f:35062 Zbl 0469. 35028

[Guo and Hong 1992] B. L. Guo and M. C. Hong, "Landau-Lifshitz equations of the ferromagnetic spin chain and harmonic maps", Adv. in Math. (China) 21:4 (1992), 501-503. MR 1204969 Zbl 0782.58053

[Guo and Hong 1993] B. L. Guo and M. C. Hong, "The Landau-Lifshitz equation of the ferromagnetic spin chain and harmonic maps", Calc. Var. Partial Differential Equations 1:3 (1993), 311-334. MR 94m:58059 Zbl 0798.35139

[Hélein 1990] F. Hélein, "Régularité des applications faiblement harmoniques entre une surface et une sphère”, C. R. Acad. Sci. Paris Sér. I Math. 311:9 (1990), 519-524. MR 92a:58034 Zbl 0728. 35014

[Joly et al. 2000] P. Joly, A. Komech, and O. Vacus, "On transitions to stationary states in a MaxwellLandau-Lifschitz-Gilbert system”, SIAM J. Math. Anal. 31:2 (2000), 346-374. MR 2001c:78006 Zbl 1025.78005

[Landau and Lifshitz 1935] L. Landau and D. Lifshitz, "On the theory of the dispersion of magnetic permeability in ferromagnetic bodies", Phys. Z. Soviet Union 8 (1935), 153-169. Zbl 0012.28501 
[Lieberman 1996] G. M. Lieberman, Second order parabolic differential equations, World Scientific, River Edge, NJ, 1996. MR 98k:35003 Zbl 0884.35001

[Liu 2004] X.-G. Liu, "Partial regularity for the Landau-Lifshitz system", Calc. Var. Partial Differential Equations 20:2 (2004), 153-173. MR 2005a:58025 Zbl 1058.58008

[Melcher 2005] C. Melcher, "Existence of partially regular solutions for Landau-Lifshitz equations in $\mathbb{R}^{3}$ ", Comm. Partial Differential Equations 30:4-6 (2005), 567-587. MR 2006i:35354 Zbl 1074.35023

[Moser 2002] R. Moser, "Partial regularity for the Landau-Lifshitz equation in small dimensions", preprint 26/2002, Max-Planck-Institut Leipzig, 2002, Available at http://www.mis.mpg.de/preprints/ 2002/preprint2002_26.pdf.

[Struwe 1988] M. Struwe, "On the evolution of harmonic maps in higher dimensions", J. Differential Geom. 28:3 (1988), 485-502. MR 90j:58037 Zbl 0631.58004

[Wang 2006] C. Wang, "On Landau-Lifshitz equation in dimensions at most four", Indiana Univ. Math. J. 55:5 (2006), 1615-1644. MR 2007h:35180 Zbl 1105.35049

[Zhou and Guo 1987] Y. L. Zhou and B. L. Guo, "Weak solution of system of ferromagnetic chain with several variables”, Sci. Sinica Ser. A 30 (1987), 1251-1266. MR 89h:35323 Zbl 0656.35123

[Zhou et al. 1991] Y. L. Zhou, B. L. Guo, and S. B. Tan, "Existence and uniqueness of smooth solution for system of ferro-magnetic chain”, Sci. China Ser. A 34:3 (1991), 257-266. MR 92i:82030 Zbl 0752.35074

Received December 4, 2008. Revised February 9, 2009.

\title{
SHIJIN DING
}

DEPARTMENT OF MATHEMATICS

SOUTH CHINA NORMAL UNIVERSITY

GUANGZHOU, GUANGDONG 510631

CHINA

dingsj@scnu.edu.cn

\author{
XiAngaO LiU \\ SCHOOL OF MATHEMATICS \\ FUDAN UNIVERSITY \\ SHANGHAI, 200433 \\ CHINA \\ xgliu@fudan.edu.cn \\ Changyou Wang \\ DePartment of Mathematics \\ UNIVERSITY OF KENTUCKY \\ LEXINGTON, KY 40506 \\ UNITED STATES \\ cywang@ms.uky.edu \\ http://www.ms.uky.edu/ cywang
}

